\title{
THE SEMIADDITIVITY OF CONTINUOUS ANALYTIC CAPACITY AND THE INNER BOUNDARY CONJECTURE
}

\author{
XAVIER TOLSA
}

\begin{abstract}
Let $\alpha(E)$ be the continuous analytic capacity of a compact set $E \subset \mathbb{C}$. In this paper we obtain a characterization of $\alpha$ in terms of curvature of measures with zero linear density, and we deduce that $\alpha$ is countably semiadditive. This result has important consequences for the theory of uniform rational approximation on compact sets. In particular, it implies the so called inner boundary conjecture.
\end{abstract}

\section{INTRODUCTION}

The continuous analytic capacity $\alpha$ was introduced by Erokhin and Vitushkin (see [Vi]) in the 1950's in order to study problems of uniform rational approximation in compact subsets of the complex plane. Up to now, the geometric properties of the capacity $\alpha$ have not been well understood. It has been an open question if $\alpha$ is semiadditive as a set function, that is to say, if

$$
\alpha(E \cup F) \leq C(\alpha(E)+\alpha(F)),
$$

for arbitrary compact sets $E, F \subset \mathbb{C}$, where $C$ is an absolute constant. It was known that an affirmative answer to this question would imply important results on uniform rational approximation, such as the so called "inner boundary conjecture" (see [VM, Conjecture 2]). In this paper we will show that $\alpha$ is indeed semiadditive. As a consequence, the inner boundary conjecture is true. Our proof of the semiadditivity of $\alpha$ does not follow from the recently proved semiaddivity of analytic capacity $\gamma$ in [To5]. However, some of the ideas and techniques of [To5] are essential for the results of this paper.

To state these results in more detail, we need to introduce some notation and terminology. The continuous analytic capacity of a compact set $E \subset \mathbb{C}$ is defined as

$$
\alpha(E)=\sup \left|f^{\prime}(\infty)\right|
$$

where the supremum is taken over all complex functions which are continuous in $\mathbb{C}$, analytic on $\mathbb{C} \backslash E$, and satisfy $|f(z)| \leq 1$ for all $z \in \mathbb{C}$; and $f^{\prime}(\infty)=\lim _{z \rightarrow \infty} z(f(z)-f(\infty))$. For a general set $F$, we set $\alpha(F)=$ $\sup \{\alpha(E): E \subset F, E$ compact $\}$.

Date: May, 2002.

Supported by the program Ramón y Cajal (Spain). Also partially supported by grants DGICYT BFM2000-0361 (Spain) and 2001/SGR/00431 (Generalitat de Catalunya). 
If in the supremum above we don't ask the continuity on $\mathbb{C}$ for $f$ (we only ask $f$ to be analytic on $\mathbb{C} \backslash E$ and $|f(z)| \leq 1$ for all $z \in \mathbb{C} \backslash E$ ), we obtain the analytic capacity $\gamma$ of the compact set $E$. For a general set $F$, we set $\gamma(F)=\sup \{\gamma(E): E \subset F, E$ compact $\}$.

Our main result is the following.

Theorem 1.1. Let $E_{i}, i \geq 1$, be Borel sets in $\mathbb{C}$. Then,

$$
\alpha\left(\bigcup_{i=1}^{\infty} E_{i}\right) \leq C \sum_{i=1}^{\infty} \alpha\left(E_{i}\right),
$$

where $C$ is an absolute constant.

The semiadditivity of continuous analytic capacity was already known in some special cases. Melnikov [Me1] proved it for two compact sets $E, F$ separated by an analytic curve. Vitushkin [Vi] extended the result to the case in which the curve which separates $E$ and $F$ is piecewise Lyapunov. Davie [Dve] showed that it is enough to assume that the curve is hypo-Lyapunov. On the other hand, Davie also proved in [Dve] that the semiadditivity of $\gamma$ for arbitrary compact sets (recently proved in [To5]) implies the semiaddivity of $\alpha$ for disjoint compact sets. However, as far as we know, the semiadditivity of $\alpha$ for arbitrary compact sets (which is needed for the proof of the inner boundary conjecture) cannot be derived directly from the semiadditivity of $\gamma$. Melnikov, Paramonov and Verdera [VMP] also proved the semiadditivity of $\alpha_{+}$(which is a variant of $\alpha$ originated by Cauchy transforms of positive measures) in several cases (in particular, for bounded Borel sets $E$ and $F$ which are relatively open in the topology of $E \cup F$ ).

We need now to introduce some additional terminology in connection with uniform rational approximation. Given $E \subset \mathbb{C}$ compact, we denote by $R(E)$ the algebra of complex functions on $E$ which are uniform limits on $E$ of functions analytic in a neighborhood of $E$ (i.e. each function is analytic in a neighborhood of $E) . A(E)$ is the algebra of those complex functions on $E$ which are continuous on $E$ and analytic on $\stackrel{\circ}{E}$. Vitushkin proved that the following result is a consequence of the semiadditivity of $\alpha$ (see [Vi, p.187]).

Theorem 1.2. Suppose that for all $z \in \partial E$, with the exception of a set with zero continuous analytic capacity, we have

$$
\limsup _{r \rightarrow 0} \frac{\alpha(\bar{B}(z, r) \backslash \stackrel{\circ}{E})}{\alpha(\bar{B}(z, r) \backslash E)}<\infty
$$

Then, $R(E)=A(E)$.

The inner boundary of $E$, denoted by $\partial_{i} E$, is the set of boundary points which do not belong to the boundary of any connected component of $\mathbb{C} \backslash E$. The inner boundary conjecture is a corollary of the preceding theorem:

Theorem 1.3 (Inner boundary conjecture). If $\alpha\left(\partial_{i} E\right)=0$, then $R(E)=$ $A(E)$. 
In the special case where $\operatorname{dim}\left(\partial_{i} E\right)<1$ (where $\operatorname{dim}$ is the Hausdorff dimension), Davie and Øksendal [D $\varnothing]$ had already proved that $R(E)=$ $A(E)$. Another partial result was obtained in [MTV2] as a straightforward consequence of the semiadditivity of $\gamma$. Namely, it was shown that $R(E)=$ $A(E)$ also holds if $\gamma\left(\partial_{i} E\right)=0$.

The semiaddivity of $\alpha$ follows from a more precise result which asserts that $\alpha$ and $\alpha_{+}$are comparable. Further, $\alpha_{+}$(and also $\alpha$, because of its comparability with $\alpha$ ) can be characterized in terms of the so called curvature of measures, or equivalently in terms of $L^{2}$ estimates for the Cauchy transform. We now proceed to define the main notions involved in these results.

Given a complex Radon measure $\nu$ on $\mathbb{C}$, the Cauchy transform of $\nu$ is

$$
\mathcal{C} \nu(z)=\int \frac{1}{\xi-z} d \nu(\xi), \quad z \notin \operatorname{supp}(\nu) .
$$

This definition does not make sense, in general, for $z \in \operatorname{supp}(\nu)$, although one can easily see that the integral above is convergent at a.e. $z \in \mathbb{C}$ (with respect to the Lebesgue measure $\mathcal{L}^{2}$ ). This is the reason why one considers the truncated Cauchy transform of $\nu$, which is defined as

$$
\mathcal{C}_{\varepsilon} \nu(z)=\int_{|\xi-z|>\varepsilon} \frac{1}{\xi-z} d \nu(\xi)
$$

for any $\varepsilon>0$ and $z \in \mathbb{C}$. Given a $\mu$-measurable function $f$ on $\mathbb{C}$ (where $\mu$ is some fixed positive Radon measure on $\mathbb{C})$, we write $\mathcal{C} f \equiv \mathcal{C}(f d \mu)$ and $\mathcal{C}_{\varepsilon} f \equiv \mathcal{C}_{\varepsilon}(f d \mu)$ for any $\varepsilon>0$. It is said that the Cauchy transform is bounded on $L^{2}(\mu)$ if the operators $\mathcal{C}_{\varepsilon}$ are bounded on $L^{2}(\mu)$ uniformly on $\varepsilon>0$.

The capacity $\alpha_{+}$of a bounded set $E \subset \mathbb{C}$ is defined as

$$
\alpha_{+}(E)=\sup \mu(E),
$$

where the supremum is taken over all positive Radon measures $\mu$ supported on $E$ such that $\mathcal{C} \mu$ is a continuous function on $\mathbb{C}$ (i.e. it coincides $\mathcal{L}^{2}$-a.e. with a continuous function on $\mathbb{C}$ ), with $\|\mathcal{C} \mu\|_{L^{\infty}(\mathbb{C})} \leq 1$. Notice that we clearly have $\alpha_{+}(E) \leq \alpha(E)$, because $(\mathcal{C} \mu)^{\prime}(\infty)=\mu(E)$.

If in the definition of $\alpha_{+}(E)$ we don't ask $\mathcal{C} \mu$ to be continuous on $\mathbb{C}$, we obtain $\gamma_{+}(E)$. That is, $\gamma_{+}(E)=\sup \mu(E)$, with the supremum taken over all positive Radon measures $\mu$ supported on $E$ such that $\|\mathcal{C} \mu\|_{L^{\infty}(\mathbb{C})} \leq 1$.

A positive Radon measure $\mu$ is said to have linear growth if there exists some constant $C$ such that $\mu(B(x, r)) \leq C r$ for all $x \in \mathbb{C}, r>0$. The linear density of $\mu$ at $x \in \mathbb{C}$ is (if it exists)

$$
\Theta_{\mu}(x)=\lim _{r \rightarrow 0} \frac{\mu(B(x, r))}{r} .
$$

Given three pairwise different points $x, y, z \in \mathbb{C}$, their Menger curvature is

$$
c(x, y, z)=\frac{1}{R(x, y, z)},
$$


where $R(x, y, z)$ is the radius of the circumference passing through $x, y, z$ (with $R(x, y, z)=\infty, c(x, y, z)=0$ if $x, y, z$ lie on a same line). If two among these points coincide, we let $c(x, y, z)=0$. For a positive Radon measure $\mu$, we set

$$
c_{\mu}^{2}(x)=\iint c(x, y, z)^{2} d \mu(y) d \mu(z),
$$

and we define the curvature of $\mu$ as

$$
c^{2}(\mu)=\int c_{\mu}^{2}(x) d \mu(x)=\iiint c(x, y, z)^{2} d \mu(x) d \mu(y) d \mu(z) .
$$

The notion of curvature of measures was introduced by Melnikov [Me2] when he was studying a discrete version of analytic capacity, and it is one of the ideas which is responsible of the big recent advances in connection with analytic capacity. On the one hand, the notion of curvature is connected to the Cauchy transform. This relationship comes from the following identity found by Melnikov and Verdera [MV] (assuming that $\mu$ has linear growth):

$$
\left\|\mathcal{C}_{\varepsilon} \mu\right\|_{L^{2}(\mu)}^{2}=\frac{1}{6} c_{\varepsilon}^{2}(\mu)+O(\mu(\mathbb{C})),
$$

where $c_{\varepsilon}^{2}(\mu)$ is an $\varepsilon$-truncated version of $c^{2}(\mu)$ (defined as in the right hand side of (1.1), but with the triple integral over $\{x, y, z \in \mathbb{C}:|x-y|, \mid y-$ $z|| x-z \mid,>\varepsilon\})$.

Next theorem contains the aforementioned characterization of $\alpha$ and $\alpha_{+}$.

Theorem 1.4. For all compact sets $E \subset \mathbb{C}$, we have

$$
\begin{aligned}
& \alpha(E) \approx \alpha_{+}(E) \\
& \quad \approx \sup \left\{\mu(E): \operatorname{supp}(\mu) \subset E, \Theta_{\mu}(x)=0 \forall x \in E, c^{2}(\mu) \leq \mu(E)\right\},
\end{aligned}
$$

with absolute constants.

The notation $A \approx B$ means that $A$ is comparable to $B$, that is to say, that there exists a positive absolute constant $C$ such that $C^{-1} A \leq B \leq C A$.

In (2.3) in next section, the reader will find another characterization of $\alpha$ and $\alpha_{+}$which involves the $L^{2}$ norm of the Cauchy transform.

Let us remark that, by the results of [To4, Section 5], it follows that the supremum in (1.3) is comparable to

$$
\begin{aligned}
\sup \{\mu(E): \operatorname{supp}(\mu) \subset E, & \Theta_{\mu}(x)=0 \forall x \in E, \\
& \left.\mu(B(x, r)) \leq r \forall x \in E, r>0, c^{2}(\mu) \leq \mu(E)\right\} .
\end{aligned}
$$

See Remark 2.2 below for more details. Notice also that the latter supremum without the condition " $\Theta_{\mu}(x)=0 \forall x \in E$ " is comparable to $\gamma_{+}(E)$ and $\gamma(E)$ (see [To4] and [To5]).

The first step for proving Theorem 1.4 consists of showing that $\alpha_{+}$is comparable to the supremum in (1.3). This is done in Section 3. The difficult inequality is $\alpha_{+}(E) \geq C^{-1} \sup \ldots$. We will derive it from the fact that if $\Theta_{\mu}(x)=0 \mu$-a.e. in $\mathbb{C}$, then the $L^{2}(\mu)$ norm of the Cauchy transform 
restricted to a ball $B(x, r)$ tends to 0 as $r \rightarrow 0$ (assuming some uniformity conditions concerning the linear density and curvature of $\mu$ ). Let us remark that the same idea was used in [To1] to prove that the $L^{2}(\mu)$ boundedness of the Cauchy transform implies the existence of principal values $\mu$-a.e. when the linear density of $\mu$ is zero.

The second step for Theorem 1.4 is the proof of the comparability between $\alpha$ and $\alpha_{+}$. A possible approach consists of proving that there exists a measure $\mu$ supported on $E$ with zero linear density, such that $\mu(E) \approx \alpha(E)$, with the Cauchy transform bounded in $L^{2}(\mu)$ with absolute constants. If we try to use arguments similar to the ones of [To5] to construct $\mu$, several difficulties arise. For example, as far as we know, $\alpha_{+}$does not admit a dual characterization such as the one of $\gamma_{+}$in [To4, Theorem 3.3]. This characterization plays a key role in the proof of $\gamma \approx \gamma_{+}$. To overcome these difficulties we have introduced in Section 4 some auxiliary capacities $\gamma^{h}$ and $\gamma_{+}^{h}$ associated to a gauge function $h$ that measures how fast $\mu(B(x, r)) / r$ tends to 0 . The comparability of $\alpha$ and $\alpha_{+}$is then a consequence of the comparability between $\gamma^{h}$ and $\gamma_{+}^{h}$ for each $h$. Some properties of $\gamma^{h}$ and $\gamma_{+}^{h}$ are shown in Sections 5 and 6. They will be used in Section 7 to show that $\gamma^{h} \approx \gamma_{+}^{h}$, by arguments analogous to the ones of the proof of $\gamma \approx \gamma_{+}$in [To5].

\section{Preliminaries}

Let $M$ be the maximal radial Hardy-Littlewood operator:

$$
M \mu(x)=\sup _{r>0} \frac{\mu(B(x, r))}{r}
$$

[if $\mu$ were a complex measure, we would replace $\mu(B(x, r))$ by $|\mu|(B(x, r))$ ],

and let $c_{\mu}(x)=\left(c_{\mu}^{2}(x)\right)^{1 / 2}$. The following potential was introduced by Verdera in [Ve2]:

$$
U_{\mu}(x):=M \mu(x)+c_{\mu}(x),
$$

We recall that $\gamma_{+}$and $\gamma$ can be characterized in terms of this potential:

$$
\gamma(E) \approx \gamma_{+}(E) \approx \sup \left\{\mu(E): \operatorname{supp}(\mu) \subset E, U_{\mu}(x) \leq 1 \forall x \in \mathbb{C}\right\} .
$$

From Theorem 1.4 (see also Remark 2.2 below) we infer that $\alpha$ and $\alpha_{+}$also can be described using the potential $U_{\mu}$ :

$\alpha(E) \approx \alpha_{+}(E) \approx \sup \left\{\mu(E): \operatorname{supp}(\mu) \subset E, \Theta_{\mu}(x)=0, U_{\mu}(x) \leq 1 \forall x \in \mathbb{C}\right\}$.

Moreover, using the identity (1.2), with arguments analogous to the ones for $\gamma_{+}$in [To2] and [To4], it can be shown that (1.3) and (2.2) are equivalent to $(2.3)$

$\alpha(E) \approx \alpha_{+}(E) \approx \sup \left\{\mu(E): \operatorname{supp}(\mu) \subset E, \Theta_{\mu}(x)=0,\|\mathcal{C}\|_{L^{2}(\mu), L^{2}(\mu)} \leq 1\right\}$. 
Given two positive Radon measure $\nu$ and $\mu$, we set

$$
c^{2}(x, \nu, \mu)=\iint c(x, y, z)^{2} d \nu(y) d \mu(z) .
$$

Remember the following maximum principle for curvature of measures (see [To2] and [To4]).

Lemma 2.1. Let $\mu$ and $\nu$ be positive Radon measures such that

$$
c^{2}(x, \nu, \mu) \leq \beta \quad \text { for all } x \in \operatorname{supp}(\nu) .
$$

Then,

$$
c^{2}(x, \nu, \mu) \leq 2 \beta+C_{1} M \nu(x) \cdot \sup _{z \in \operatorname{supp}(\nu)} M \mu(z) \quad \text { for all } x \in \mathbb{C} .
$$

If $c_{\mu}^{2}(x) \leq \beta$ for all $x \in \operatorname{supp}(\mu)$, then

$$
c_{\mu}^{2}(x) \leq 2 \beta+C_{1} M \mu(x)^{2} \quad \text { for all } x \in \mathbb{C} .
$$

Remark 2.2. If $\mu$ is such that $\Theta_{\mu}^{*}(x):=\limsup _{r \rightarrow 0} \mu(B(x, r)) / r \leq 1$ and $c_{\mu}(x) \leq 1$ for all $x \in \operatorname{supp}(\mu)$, then there exists some absolute constant $C$ such that $U_{\mu}(x) \leq C$ for all $x \in \mathbb{C}$. This follows from Lemma 5.2 in [To4], which asserts that if $\nu$ is a positive Radon measure supported on $B\left(x_{0}, R\right)$ with $\Theta_{\nu}^{*}(x) \leq 1 \nu$-a.e. and $c^{2}(\nu) \leq \nu\left(B\left(x_{0}, R\right)\right)$, then there is some absolute constant $M_{0}$ such that $\nu\left(B\left(x_{0}, R\right)\right) \leq M_{0} R$. Indeed, from the condition

$$
c_{\mu}(x) \leq 1 \quad \text { for all } x \in \operatorname{supp}(\mu)
$$

we deduce

$$
c^{2}(\mu \mid B(x, r)) \leq \mu(B(x, r)) \quad \text { for all } x \in \operatorname{supp}(\mu), r>0,
$$

and so $M \mu(x) \leq M_{0}$ for all $x \in \operatorname{supp}(\mu)$. Thus $U_{\mu}(x) \leq M_{0}+1$ for all $x \in \operatorname{supp}(\mu)$, and then, by the maximum principle above, $U_{\mu}(x) \leq C$ for all $x \in \mathbb{C}$.

This is the reason why the statement " $\mu(B(x, r)) \leq r$ " can be avoided in (1.3).

Let us recall the definition of the maximal Cauchy transform of a complex measure $\nu$ :

$$
\mathcal{C}_{*} \nu(x)=\sup _{\varepsilon>0}\left|\mathcal{C}_{\varepsilon} \nu(x)\right| .
$$

If $f$ is a $\mu$-measurable function (for a fixed measure $\mu$ ), we set $\mathcal{C}_{*} f \equiv$ $\mathcal{C}_{*}(f d \mu)$.

Let $\psi$ be a $\mathcal{C}^{\infty}$ radial function supported on $B(0,1)$, with $0 \leq \psi \leq 2$, $\|\nabla \psi\|_{\infty} \leq 10$, and $\int \psi d \mathcal{L}^{2}=1$ (where $\mathcal{L}^{2}$ stands for the Lebesgue measure). We denote $\psi_{\varepsilon}(x)=\varepsilon^{-2} \psi(x / \varepsilon)$. The regularized operators $K_{\varepsilon}$ are defined as

$$
K_{\varepsilon} \nu:=\psi_{\varepsilon} * \mathcal{C} \nu=\psi_{\varepsilon} * \frac{1}{z} * \nu
$$


Let $k_{\varepsilon}=\psi_{\varepsilon} * \frac{1}{z}$. It is easily seen that $k_{\varepsilon}(z)=1 / z$ if $|z|>\varepsilon,\left\|k_{\varepsilon}\right\|_{\infty} \leq C / \varepsilon$, and $\left|\nabla k_{\varepsilon}(z)\right| \leq C|z|^{-2}$. Further, since $k_{\varepsilon}$ is a uniformly continuous kernel, $K_{\varepsilon} \nu$ is a continuous function on $\mathbb{C}$.

Notice that if $|\mathcal{C} \nu| \leq B$ a. e. with respect to Lebesgue measure, then $\left|K_{\varepsilon} \nu(z)\right| \leq B$ for all $z \in \mathbb{C}$. Moreover, we have

$$
\left|K_{\varepsilon} \nu(x)-\mathcal{C}_{\varepsilon} \nu(x)\right|=\left|\int_{|y-x| \leq \varepsilon} k_{\varepsilon}(y-x) d \nu(y)\right| \leq C M \nu(x) .
$$

We will also use the notation $k_{\varepsilon}(x, y):=k_{\varepsilon}(y-x)$.

The corresponding maximal operator associated to $K_{\varepsilon}$ is

$$
K_{*} \nu(x)=\sup _{\varepsilon>0}\left|K_{\varepsilon} \nu(x)\right| .
$$

Given a $\mu$-measurable function $f$ on $\mathbb{C}$, we write $K_{\varepsilon} f \equiv K_{\varepsilon}(f d \mu)$ and $K_{*} f \equiv$ $K_{*}(f d \mu)$ for any $\varepsilon>0$.

Observe that the definitions of $K_{\varepsilon} \nu$ and $K_{*} \nu$ also make sense if $\nu$ is a distribution with compact support instead of a measure.

By a square (rectangle) $Q$ we mean a closed square (rectangle) with sides parallel to the axes, unless otherwise stated.

Throughout all the paper, the letter $C$ will stand for an absolute constant that may change at different occurrences. Constants with subscripts, such as $C_{1}$, will retain its value, in general.

\section{THE CAPACITY $\alpha_{+}$}

In this section we will prove:

Theorem 3.1. For all compact sets $E \subset \mathbb{C}$, we have

$\alpha_{+}(E) \approx \sup \left\{\mu(E): \operatorname{supp}(\mu) \subset E, \Theta_{\mu}(x)=0 \forall x \in E, U_{\mu}(x) \leq 1 \forall x \in \mathbb{C}\right\}$, with absolute constants.

We denote

$\alpha_{0}(E):=\sup \left\{\mu(E): \operatorname{supp}(\mu) \subset E, \Theta_{\mu}(x)=0 \forall x \in E, U_{\mu}(x) \leq 1 \forall x \in \mathbb{C}\right\}$.

Next lemma deals with the easy inequality of Theorem 3.1.

Lemma 3.2. There exists an absolute constant $C$ such that

$$
\alpha_{+}(E) \leq C \alpha_{0}(E)
$$

for any compact set $E \subset \mathbb{C}$.

Proof. Let $\mu$ be a Radon measure supported on $E$ such that $\mathcal{C} \mu$ is a continuous function on $\mathbb{C}$, with $\|\mathcal{C} \mu\|_{L^{\infty}(\mathbb{C})} \leq 1$, and $\mu(E) \geq \alpha_{+}(E) / 2$. Then, for al $x \in \mathbb{C}$ and $r>0$ we have

$$
\mu(B(x, r))=\frac{-1}{2 \pi i} \int_{|z-x|=r} \mathcal{C} \mu(z) d z \leq r
$$


(see [Gar, p.40], for example). Also,

$$
c^{2}(\mu) \leq C_{2} \mu(E)
$$

by (1.2). By Chebishev, there exists some compact set $F \subset E$ such that $c_{\mu}^{2}(x) \leq 2 C_{2}$ and $\mu(F) \geq 4 \mu(E)$. We set $\nu=\mu_{\mid F}$. Since $c_{\nu}^{2}(x) \leq 2 C_{2}$ for all $x \in F$, by the maximum principle for curvature of measures (see Lemma 2.1 ), we get

$$
c_{\nu}^{2}(x) \leq C_{3} \quad \text { for all } x \in \mathbb{C} .
$$

Thus,

$$
U_{\nu}(x) \leq 1+C_{3}^{1 / 2}=: C_{4} \text { for all } x \in \mathbb{C} .
$$

To see that $\Theta_{\nu}(x)=0$ for all $x \in E$ it suffices to show that the same condition holds for $\mu$. Given $\varepsilon>0$, let $\delta>0$ be such that $|\mathcal{C} \mu(z)-\mathcal{C} \mu(x)| \leq \varepsilon$ for $x, z \in \mathbb{C}$ with $|z-x| \leq \delta$. For $r \leq \delta$ we deduce

$$
\begin{aligned}
\mu(B(x, r)) & =\frac{-1}{2 \pi i} \int_{|z-x|=r} \mathcal{C} \mu(z) d z \\
& =\frac{-1}{2 \pi i} \int_{|z-x|=r}(\mathcal{C} \mu(z)-\mathcal{C} \mu(x)) d z \leq \varepsilon r
\end{aligned}
$$

That is, $\mu(B(x, r)) / r \leq \varepsilon$ if $r \leq \delta$, and so $\Theta_{\mu}(x)=0$.

Therefore, we have

$$
\alpha_{+}(E) \leq C \nu(E) \leq C \alpha_{0}(E)
$$

The proof of the inequality $\alpha_{0}(E) \leq C \alpha_{+}(E)$ involves the regularized operators $K_{\varepsilon}$ introduced in Section 2. The following lemma concentrates the main technical difficulties of the proof.

Lemma 3.3. Suppose that $\lim _{r \rightarrow 0} \mu(B(x, r)) / r=0$ and $\lim _{r \rightarrow 0} c_{\mu \mid B(x, r)}^{2}(x)=$ 0 both uniformly on $E$. Let $f$ be a positive bounded function supported on $E$, such that $\left\|K_{*} f\right\|_{L^{\infty}(\mathbb{C})}<\infty$. Given any $\tau>0$, there exists some $\delta>0$ and some function $g$ supported on $E$ such that $\int g d \mu=\int f d \mu$, $0 \leq g \leq\|f\|_{L^{\infty}(\mu)}+\tau,\left\|K_{*} g\right\|_{L^{\infty}(\mathbb{C})} \leq\left\|K_{*} f\right\|_{L^{\infty}(\mathbb{C})}+\tau$, and moreover

$$
\left|K_{\varepsilon} g(x)-K_{\varepsilon} g(y)\right| \leq \tau \quad \text { for }|x-y| \leq \delta \text { and all } \varepsilon>0
$$

and

$$
\left|K_{\varepsilon} g(x)-K_{\varepsilon} g(y)\right| \leq \sup _{\varepsilon^{\prime}>0}\left|K_{\varepsilon^{\prime}} f(x)-K_{\varepsilon^{\prime}} f(y)\right|+\tau \quad \text { for all } x, y \in \mathbb{C}, \varepsilon>0 .
$$

In a sense, this lemma asserts that under the appropriate assumptions, given a positive bounded function $f$ such that $K_{*} f$ is bounded, one can construct another positive bounded function $g$ with $\int g d \mu=\int f d \mu$, with the $L^{\infty}$ norms of $g$ and $K_{*} g$ very close to the corresponding $L^{\infty}$ norms of $f$ and $K_{*} f$, such that the term $\left|K_{\varepsilon} g(x)-K_{\varepsilon} g(y)\right|$ is very small when $x$ and $y$ are close enough (by (3.2)), and such that the same term is also under control if we don't assume $x$ and $y$ to be close (by (3.3)). 
Before proving Lemma 3.3, we will show how one can deduce the inequality $\alpha_{0}(E) \leq C \alpha_{+}(E)$ from it.

Lemma 3.4. There exists an absolute constant $C$ such that

$$
\alpha_{0}(E) \leq C \alpha_{+}(E)
$$

for any compact set $E \subset \mathbb{C}$.

Proof. Let $\mu$ be a Radon measure supported on $E$ such that $\mu(E) \geq \alpha_{0}(E) / 2$, with $\Theta_{\mu}(x)=0$ for all $x \in E$, and $U_{\mu}(x) \leq 1$ for all $x \in \mathbb{C}$. Let $F \subset E$ a compact set such that $\lim _{r \rightarrow 0} \mu(B(x, r)) / r=0$ and $\lim _{r \rightarrow 0} c_{\mu \mid B(x, r)}^{2}(x)=0$ both uniformly on $F$, with $\mu(F) \geq \mu(E) / 2$. Since the Cauchy transform is bounded on $L^{2}(\mu)$, there exists a function $f_{1}$ supported on $F$, with $0 \leq f_{1} \leq 1,\left\|K_{*} f_{1}\right\|_{L^{\infty}(\mathbb{C})} \leq 1$, and $\int f_{1} d \mu \geq C^{-1} \mu(F)$. We set $\delta_{1}=1$.

For $n \geq 1$, we set $\tau_{n}=2^{-n}$, and given a positive bounded function $f_{n}$ supported on $F$ and $\delta_{n}>0$, by means of Lemma 3.3 we construct a function $f_{n+1}$ also supported on $F$, so that $\int f_{n+1} d \mu=\int f_{n} d \mu, 0 \leq f_{n+1} \leq$ $\left\|f_{n}\right\|_{L^{\infty}(\mu)}+\tau_{n},\left\|K_{*} f_{n+1}\right\|_{L^{\infty}(\mathbb{C})} \leq\left\|K_{*} f_{n}\right\|_{L^{\infty}(\mathbb{C})}+\tau_{n}$, and moreover

$$
\left|K_{\varepsilon} f_{n+1}(x)-K_{\varepsilon} f_{n+1}(y)\right| \leq \tau_{n} \text { for }|x-y| \leq \delta_{n+1} \text { and all } \varepsilon>0
$$

(where $\delta_{n+1} \leq \delta_{n}$ is some constant small enough), and

$$
\left|K_{\varepsilon} f_{n+1}(x)-K_{\varepsilon} f_{n+1}(y)\right| \leq \sup _{\varepsilon^{\prime}>0}\left|K_{\varepsilon^{\prime}} f_{n}(x)-K_{\varepsilon^{\prime}} f_{n}(y)\right|+\tau_{n}
$$

for all $x, y \in \mathbb{C}, \varepsilon>0$.

Let $f$ be a weak $*$ limit in $L^{\infty}(\mu)$ of a subsequence $\left\{f_{n_{k}}\right\}_{k}$. Clearly, $f$ is a positive bounded function such that

$$
\int f d \mu=\int f_{1} d \mu \geq C^{-1} \alpha_{0}(E) .
$$

Also, for each $\varepsilon>0$ and $x \in \mathbb{C}$,

$$
K_{\varepsilon} f_{n_{k}}(x) \rightarrow K_{\varepsilon} f(x) \quad \text { as } \quad k \rightarrow \infty .
$$

Since

$$
\left\|K_{\varepsilon} f_{n}\right\|_{L^{\infty}(\mathbb{C})} \leq\left\|K_{*} f_{1}\right\|_{L^{\infty}(\mathbb{C})}+\sum_{j=1}^{n-1} 2^{-j} \leq 2
$$

for all $n$, we deduce $\left\|K_{\varepsilon} f\right\|_{L^{\infty}(\mathbb{C})} \leq 2$.

On the other hand, by (3.4), if $|x-y| \leq \delta_{n}$, then

$$
\left|K_{\varepsilon} f_{n}(x)-K_{\varepsilon} f_{n}(y)\right| \leq 2^{-n}
$$

for all $\varepsilon>0$. From (3.5), for $k \geq n$ we get

$$
\left|K_{\varepsilon} f_{k}(x)-K_{\varepsilon} f_{k}(y)\right| \leq \sup _{\varepsilon^{\prime}>0}\left|K_{\varepsilon^{\prime}} f_{n}(x)-K_{\varepsilon^{\prime}} f_{n}(y)\right|+\sum_{j=n}^{k-1} 2^{-k} \leq 2^{-n+2},
$$

assuming $|x-y| \leq \delta_{n}$. Thus,

$$
\left|K_{\varepsilon} f(x)-K_{\varepsilon} f(y)\right| \leq 2^{-n+2} \quad \text { if }|x-y| \leq \delta_{n} .
$$


Consider now the family of functions $\left\{K_{\varepsilon} f\right\}_{\varepsilon>0}$ on $\bar{B}(0, R)$, where $R$ is big enough so that $E \subset B(0, R-1)$. This is a family of functions which is uniformly bounded and equicontinuous on $\bar{B}(0, R)$, by (3.6). By the AscoliArzelà theorem, there exists a sequence $\left\{\varepsilon_{n}\right\}_{n}$, with $\varepsilon_{n} \rightarrow 0$, such that $K_{\varepsilon_{n}} f$ converges uniformly on $\bar{B}(0, R)$ to some continuous function $g$. It is easily seen that $g$ coincides with $\mathcal{C} f \mathcal{L}^{2}$-a.e. in $\bar{B}(0, R)$ and $\|g\|_{L^{\infty}(\bar{B}(0, R))} \leq 2$. Since $\mathcal{C} f$ is also continuous in $\mathbb{C} \backslash \bar{B}(0, R)$, we deduce that $\mathcal{C} f$ can extended continuously to the whole complex plane. Therefore,

$$
\alpha_{+}(E) \geq C^{-1} \int f d \mu \geq C^{-1} \alpha_{0}(E) .
$$

To prove Lemma 3.3 we will need some preliminary results.

Lemma 3.5. Suppose that $\mu(B(x, r)) \leq C_{0} r$ for all $r>r_{0}$. Then,

$$
\int_{|x-y| \geq r_{0}} \frac{1}{|x-y|^{2}} d \mu(y) \leq C_{5} \frac{C_{0}}{r_{0}},
$$

where $C_{5}$ is an absolute constant.

The proof of this standard estimate follows easily if we split the complex plane in rings centered at $x$, for example.

Next lemma deals with a now quite well known dualization of the usual weak $(1,1)$ inequality for the Cauchy transform, which is a consequence of its $L^{2}(\mu)$ boundedness (see either [To2], [NTV1] or [To3], for example).

Lemma 3.6. Suppose that $\mu(B(x, r)) \leq \varepsilon_{0} r$ for all $x \in \mathbb{C}, r>0$, and $\|\mathcal{C}\|_{L^{2}(\mu), L^{2}(\mu)} \leq \varepsilon_{0}$. Given $\tau>0$, for all $F \subset \mathbb{C}$ there exists some function $\varphi$ supported on $F$, such that $0 \leq \varphi \leq 1, \int \varphi d \mu \geq(1+\tau)^{-1} \mu(F)$, and $\left\|K_{\varepsilon} \varphi\right\|_{L^{\infty}(\mu)} \leq C\left(\tau, \varepsilon_{0}\right)$ for all $\varepsilon>0$. Moreover, for any fixed $\tau>0$, $C\left(\tau, \varepsilon_{0}\right) \rightarrow 0$ as $\varepsilon_{0} \rightarrow 0$.

For the proof, see [Ch, p.107-108]. Regarding the fact that $\varphi$ can be chosen independently of $\varepsilon$, an argument similar to the one in [To2, Lemma 4.4], for example, works.

Proof of Lemma 3.3. Consider the grid of all dyadic squares $\left\{Q_{i}\right\}_{i \in I}$ with side length $\ell$. Notice that since $\Theta_{\mu}(x)=0$ for all $x$, we have $\mu\left(\partial Q_{i}\right)=0$ for all $i$. Let $N$ be some big integer and $\varepsilon_{1} \ll \tau /\|f\|_{L^{\infty}(\mu)}$ some small constant both to be chosen later. We will take $\ell$ small enough so that

$$
\frac{\mu(B(x, r))}{r} \leq \varepsilon_{1} \quad \text { for all } x \in E, 0<r \leq N \ell .
$$

We also assume that $\ell$ has been chosen so small that

$$
c_{\mu \mid Q_{i}}(x) \leq \varepsilon_{1} \quad \text { for all } x \in E \cap Q_{i} .
$$

Notice that (3.7) and (3.8) imply that

$$
\|\mathcal{C}\|_{L^{2}\left(\mu \mid Q_{i}\right), L^{2}\left(\mu \mid Q_{i}\right)} \leq \varepsilon_{2},
$$


with $\varepsilon_{2} \rightarrow 0$ as $\varepsilon_{1} \rightarrow 0$ (using Theorem 1.1 of [To2], for example). This will be the main ingredient of the proof.

Now we are going to start the construction of $g$. Let $Q_{i}$ be some fixed square of the grid such that $\mu\left(Q_{i}\right) \neq 0$. Since the Cauchy transform is bounded on $L^{2}\left(\mu \mid Q_{i}\right)$ with a very small norm (assuming $\varepsilon_{2}$ very small), by Lemma 3.6 there exists some function $\varphi_{i}$ supported on $Q_{i}$ (not depending on $\varepsilon$ ), with $0 \leq \varphi_{i} \leq 1$, such that

$$
\int \varphi_{i} d \mu \geq(1+\tau)^{-1} \mu\left(Q_{i}\right)
$$

and

$$
\left\|K_{\varepsilon} \varphi_{i}\right\|_{L^{\infty}(\mathbb{C})} \leq \varepsilon_{3},
$$

with $\varepsilon_{3} \rightarrow 0$ as $\|\mathcal{C}\|_{L^{2}\left(\mu \mid Q_{i}\right)} \rightarrow 0$. We set

$$
g_{i}=\frac{\int_{Q_{i}} f d \mu}{\int \varphi_{i} d \mu} \varphi_{i}
$$

Then, by (3.9), we have

$$
\left\|K_{\varepsilon} g_{i}\right\|_{L^{\infty}(\mathbb{C})} \leq \frac{\int_{Q_{i}} f d \mu}{\int \varphi_{i} d \mu} \varepsilon_{3} \leq \varepsilon_{3}(1+\tau)\|f\|_{L^{\infty}(\mu)} \leq 2 \varepsilon_{3}\|f\|_{L^{\infty}(\mu)}
$$

(assuming $\tau \leq 1$ ). We denote $g=\sum_{i \in I} g_{i}$. By construction $g \geq 0, \int g d \mu=$ $\int f d \mu$ and, by (3.9),

$$
\|g\|_{L^{\infty}(\mu)}=\sup _{i \in I}\left\|g_{i}\right\|_{L^{\infty}(\mu)} \leq(1+\tau)\|f\|_{L^{\infty}(\mu)} .
$$

Proof of (3.2). We set $\delta=\ell / 2$. Let $Q_{i}$ be some fixed square of the grid. Notice that if $x \in Q_{i}$ and $|x-y| \leq \delta$, then $y \in 2 Q_{i}$. We set $g_{a}=g \chi_{3 Q_{i}}$ and $g_{b}=g \chi_{\mathbb{C} \backslash 3 Q_{i}}$.

First we deal with $g_{a}$. We have

$$
\begin{aligned}
\left|K_{\varepsilon} g_{a}(x)-K_{\varepsilon} g_{a}(y)\right| & \leq\left|K_{\varepsilon} g_{a}(x)\right|+\left|K_{\varepsilon} g_{a}(y)\right| \\
(3.11) & \leq \sum_{k: Q_{k} \subset 3 Q_{i}}\left(\left|K_{\varepsilon} g_{k}(x)\right|+\left|K_{\varepsilon} g_{k}(y)\right|\right) \leq 36 \varepsilon_{3}\|f\|_{L^{\infty}(\mu)}
\end{aligned}
$$

Now we consider the term originated by $g_{b}$. We have

$$
\begin{aligned}
\left|K_{\varepsilon} g_{b}(x)-K_{\varepsilon} g_{b}(y)\right| & \leq\|g\|_{L^{\infty}(\mu)} \int_{\mathbb{C} \backslash 3 Q_{i}}\left|k_{\varepsilon}(x, z)-k_{\varepsilon}(y, z)\right| d \mu(z) \\
& \leq C \ell\|f\|_{L^{\infty}(\mu)} \int_{\mathbb{C} \backslash 3 Q_{i}} \frac{1}{|x-z|^{2}} d \mu(z) .
\end{aligned}
$$


To estimate the last integral we use that the ratio $\mu(B(x, r)) / r$ is very small if $r \leq N \ell$. Indeed, by Lemma 3.5 and (3.7) we get

$$
\begin{aligned}
\int_{\mathbb{C} \backslash 3 Q_{i}} \frac{1}{|x-z|^{2}} d \mu(z) & \leq\left(\int_{\ell \leq|x-z| \leq N \ell}+\int_{|x-z|>N \ell}\right) \frac{1}{|x-z|^{2}} d \mu(z) \\
& \leq C_{5}\left(\frac{\varepsilon_{1}}{\ell}+\frac{1}{N \ell}\right) .
\end{aligned}
$$

Therefore, if $\varepsilon_{1}$ is small enough and $N$ big enough, then

$$
\left|K_{\varepsilon} g_{b}(x)-K_{\varepsilon} g_{b}(y)\right| \leq \frac{1}{2} \tau .
$$

Thus, from the preceding estimate and (3.11), we deduce

$$
\left|K_{\varepsilon} g(x)-K_{\varepsilon} g(y)\right| \leq \tau,
$$

assuming that $\varepsilon_{3} \ll \tau /\|f\|_{L^{\infty}(\mu)}$.

Proof of (3.3). Suppose now that $x \in Q_{i}$ and $y \in Q_{j}$. We write

$$
\begin{aligned}
\left|K_{\varepsilon} g(x)-K_{\varepsilon} g(y)\right| \leq \mid & \left|K_{\varepsilon}\left(g \chi_{\mathbb{C} \backslash 3 Q_{i}}\right)(x)-K_{\varepsilon}\left(g \chi_{\mathbb{C} \backslash 3 Q_{j}}\right)(y)\right| \\
& +\left|K_{\varepsilon}\left(g \chi_{3 Q_{i}}\right)(x)\right|+\left|K_{\varepsilon}\left(g \chi_{3 Q_{j}}\right)(y)\right| .
\end{aligned}
$$

By (3.10), each one of the last two terms is bounded above by $18 \varepsilon_{3}\|f\|_{L^{\infty}(\mu)} \leq$ $\tau / 10$. So it only remains to estimate the first term on the right hand side. We set

$$
\begin{aligned}
\left|K_{\varepsilon}\left(g \chi_{\mathbb{C} \backslash 3 Q_{i}}\right)(x)-K_{\varepsilon}\left(g \chi_{\mathbb{C} \backslash 3 Q_{j}}\right)(y)\right| & \leq\left|K_{\varepsilon}\left(f \chi_{\mathbb{C} \backslash 3 Q_{i}}\right)(x)-K_{\varepsilon}\left(f \chi_{\mathbb{C} \backslash 3 Q_{j}}\right)(y)\right| \\
& +\left|K_{\varepsilon}\left(g \chi_{\mathbb{C} \backslash 3 Q_{i}}\right)(x)-K_{\varepsilon}\left(f \chi_{\mathbb{C} \backslash 3 Q_{i}}\right)(x)\right| \\
& +\left|K_{\varepsilon}\left(g \chi_{\mathbb{C} \backslash 3 Q_{j}}\right)(y)-K_{\varepsilon}\left(f \chi_{\mathbb{C} \backslash 3 Q_{j}}\right)(y)\right| \\
& =I+I I+I I I .
\end{aligned}
$$

Let us estimate $I$. We set $\varepsilon^{\prime}=\max (\varepsilon, \ell)$. First we are going to show that $K_{\varepsilon}\left(f \chi_{\mathbb{C} \backslash 3 Q_{i}}\right)(x)$ is very close to $K_{\varepsilon^{\prime}} f(x)$. We have

$$
\begin{aligned}
& \left|K_{\varepsilon}\left(f \chi_{\mathbb{C} \backslash 3 Q_{i}}\right)(x)-K_{\varepsilon^{\prime}} f(x)\right| \\
& \leq\|f\|_{L^{\infty}(\mu)} \int\left|k_{\varepsilon^{\prime}}(x, z)-k_{\varepsilon}(x, z) \chi_{\mathbb{C} \backslash 3 Q_{i}}(z)\right| d \mu(z) .
\end{aligned}
$$

If $\varepsilon \geq \ell$, then $\varepsilon^{\prime}=\varepsilon$, and so the integral above equals

$$
\int_{3 Q_{i}}\left|k_{\varepsilon}(x, z)\right| d \mu(z) \leq \frac{\mu\left(3 Q_{i}\right)}{\ell} \leq C \varepsilon_{1} .
$$

If $\varepsilon<\ell$, then $k_{\varepsilon^{\prime}}(x, z)=k_{\varepsilon}(x, z)$ for $z \notin 3 Q_{i}$, and so the last integral in (3.14) now equals

$$
\int_{3 Q_{i}}\left|k_{\varepsilon^{\prime}}(x, z)\right| d \mu(z) \leq C \frac{\mu\left(3 Q_{i}\right)}{\ell} \leq C \varepsilon_{1} .
$$

Thus in any case we have

$$
\left|K_{\varepsilon}\left(f \chi_{\mathbb{C} \backslash 3 Q_{i}}\right)(x)-K_{\varepsilon^{\prime}} f(x)\right| \leq C \varepsilon_{1}\|f\|_{L^{\infty}(\mu)} .
$$


The same estimate holds interchanging $Q_{i}$ by $Q_{j}$ and $x$ by $y$. Therefore,

$$
I \leq \sup _{\varepsilon^{\prime}>0}\left|K_{\varepsilon^{\prime}} f(x)-K_{\varepsilon^{\prime}} f(y)\right|+\tau / 4,
$$

since $\varepsilon_{1} \ll \tau /\|f\|_{L^{\infty}(\mu)}$.

Let us turn our attention to $I I$. We have

$$
\begin{aligned}
I I & =\left|\int_{\mathbb{C} \backslash 3 Q_{i}} k_{\varepsilon}(x, z)[g(z)-f(z)] d \mu(z)\right| \\
& \leq \sum_{k=1}^{\infty}\left|\int_{3^{k+1} \backslash 3^{k} Q_{i}} k_{\varepsilon}(x, z)[g(z)-f(z)] d \mu(z)\right|=: \sum_{k=1}^{\infty} A_{k} .
\end{aligned}
$$

We denote by $z_{h}$ the center of each square $Q_{h}$. Then we get

$$
\begin{aligned}
A_{k} & \leq \sum_{h \in I: Q_{h} \subset 3^{k+1} Q_{i} \backslash 3^{k} Q_{i}}\left|\int_{Q_{h}} k_{\varepsilon}(x, z)[g(z)-f(z)] d \mu(z)\right| \\
& \leq \sum_{h \in I: Q_{h} \subset 3^{k+1} Q_{i} \backslash 3^{k} Q_{i}} \int_{Q_{h}}\left|k_{\varepsilon}(x, z)-k\left(x, z_{h}\right)\right||g(z)-f(z)| d \mu(z) \\
& \leq C \sum_{h \in I: Q_{h} \subset 3^{k+1} Q_{i} \backslash 3^{k} Q_{i}} \frac{\ell}{\left(3^{k} \ell\right)^{2}} \mu\left(Q_{h}\right)\|f\|_{L^{\infty}(\mu)} \\
& \leq C \frac{\mu\left(3^{k+1} Q_{i}\right)}{3^{k} \ell\left(3^{k+1} Q_{i}\right)}\|f\|_{L^{\infty}(\mu)} .
\end{aligned}
$$

Therefore, assuming that we take $N:=3^{M}$, where $M$ is some big integer, we get

$$
\begin{aligned}
I I & \leq C\left(\sum_{k=1}^{M} 3^{-k} \varepsilon_{1}+C \sum_{k=M+1}^{\infty} 3^{-k}\right)\|f\|_{L^{\infty}(\mu)} \\
& \leq C\left(\varepsilon_{1}+\frac{1}{N}\right)\|f\|_{L^{\infty}(\mu)} .
\end{aligned}
$$

Thus, $I I \leq \tau / 4$ if $\varepsilon_{1}$ and $N$ have been chosen appropriately.

The term $I I I$ is estimated in the same way as $I I$, and so we also have $I I I \leq \tau / 4$. Now, gathering the estimates corresponding to $I, I I, I I I$ and to the last two terms in (3.12), we obtain (3.3).

Proof of $\left\|K_{*} g\right\|_{L^{\infty}(\mathbb{C})} \leq\left\|K_{*} f\right\|_{L^{\infty}(\mathbb{C})}+\tau$. For $x \in Q_{i}$, we have

$$
\begin{aligned}
\left|K_{\varepsilon} g(x)\right| \leq & \left|K_{\varepsilon}\left(g \chi_{3 Q_{i}}\right)(x)\right|+\left|K_{\varepsilon}\left(g \chi_{\mathbb{C} \backslash 3 Q_{i}}\right)(x)-K_{\varepsilon}\left(f \chi_{\mathbb{C} \backslash 3 Q_{i}}\right)(x)\right| \\
& +K_{*}\left(f \chi_{\mathbb{C} \backslash 3 Q_{i}}\right)(x) .
\end{aligned}
$$

By (3.10), the first term is bounded above by $18 \varepsilon_{3}\|f\|_{L^{\infty}(\mu)} \leq \tau / 10$. The second one is estimated like the term $I I$ in (3.13).

From Theorem 3.1 and the characterization of $\gamma$ in terms of curvature of measures, we deduce the following result. 
Corollary 3.7. Let $E \subset \mathbb{C}$ be compact. Suppose that $\gamma(F)=0$ for any compact set $F \subset E$ with $\mathcal{H}^{1}(F)<\infty$. Then, $\alpha(E) \approx \gamma(E)$.

Proof. Let $\mu$ be supported on $E$ such that $\mu(E) \approx \gamma(E)$ and $U_{\mu}(x) \leq 1$ for all $x \in \mathbb{C}$. Let

$$
E_{n}=\left\{x \in E: \Theta_{\mu}^{*}(x)>1 / n\right\} .
$$

It is easily seen that $\mathcal{H}^{1}\left(E_{n}\right)<\infty$. Since $\gamma\left(E_{n}\right)=0$, we have $\mu\left(E_{n}\right)=0$. Thus, $\Theta(x)=0$ for $\mu$-a.e $x \in E$. By Theorem 3.1, this implies that $\alpha_{+}(E) \geq$ $C^{-1} \mu(E) \approx \gamma(E)$, and so $\alpha(E) \approx \gamma(E)$.

\section{The CAPACITIES $\gamma^{h}$ AND $\gamma_{+}^{h}$}

Let us define the capacity $\gamma^{h}$ of a compact set $E \subset \mathbb{C}$. We consider a continuous function $h:(0,+\infty) \longrightarrow(0,+\infty)$ such that $h(r) / r$ is non decreasing in $r$,

$$
h(r) \leq r \quad \text { and } \quad h(2 r) \leq 4 h(r) \quad \text { for all } r>0,
$$

and moreover

$$
\lim _{r \rightarrow 0+} \frac{h(r)}{r}=0
$$

We set

$$
\gamma^{h}(E)=\sup \left|f^{\prime}(\infty)\right|,
$$

where the supremum is taken over all functions $f \in L^{\infty}(\mathbb{C})$ which are analytic in $\mathbb{C} \backslash E$, with $f(\infty)=0,\|f\|_{L^{\infty}(\mathbb{C})} \leq 1$, such that

$$
\left|\int f \bar{\partial} \varphi d \mathcal{L}^{2}\right| \leq h(r) r\|\nabla \varphi\|_{\infty}
$$

for any real function $\varphi \in \mathcal{C}_{c}^{\infty}$ supported on some ball of radius $r$. If $f$ satisfies all these properties we say that $f$ is admissible for $\gamma^{h}$ and $E$, and we write $f \in A^{h}(E)$.

The capacity $\gamma_{+}^{h}(E)$ is defined in an analogous way, but we ask an additional condition on the functions in the supremum above. Namely, $f$ must be the Cauchy transform of some positive Radon measure supported on $E$. We say that $f$ is admissible for $\gamma_{+}^{h}$ and $E$, and we write $f \in A_{+}^{h}(E)$.

Let us remark that the doubling property $h(2 r) \leq 4 h(r)$, for $r>0$, implies that

$$
h(\lambda t) \leq 4 \lambda^{2} h(t) \quad \text { for all } \lambda>1 \text { and } t>0 .
$$

Lemma 4.1. There exists an absolute constant $C$ such that for any compact set $E$,

$$
\alpha(E) \leq C \sup _{h} \gamma^{h}(E),
$$

with the supremum over all continuous functions $h:(0,+\infty) \longrightarrow(0,+\infty)$ satisfying (4.1) and (4.2), with $h(r) / r$ non decreasing. 
Proof. Let $f$ be a continuous function on $\mathbb{C}$, analytic in $\mathbb{C} \backslash E$, such that $\|f\|_{L^{\infty}(\mathbb{C})} \leq 1$, and $\left|f^{\prime}(\infty)\right| \geq \alpha(E) / 2$. Let $w_{f}(r)$ be the modulus of continuity of $f$, that is

$$
w_{f}(r):=\sup _{x, y:|x-y| \leq r}|f(x)-f(y)| .
$$

Notice that $\left|w_{f}(r)\right| \leq 2$ for all $r>0, w_{f}$ is continuous, non decreasing, and by the triangle inequality $w_{f}(2 r) \leq 2 w_{f}(r)$. We set $h(r)=r w_{f}(r) / 2$, and so $h$ fulfills the properties (4.1) and (4.2). Then, if $\varphi$ is a real $\mathcal{C}^{\infty}$ function supported on $B\left(x_{0}, r\right)$, we have

$$
\begin{aligned}
\left|\int f \bar{\partial} \varphi d \mathcal{L}^{2}\right| & =\left|\int\left(f(x)-f\left(x_{0}\right)\right) \bar{\partial} \varphi(x) d \mathcal{L}^{2}(x)\right| \\
& \leq \pi r^{2} w_{f}(r)\|\nabla \varphi\|_{\infty}=2 \pi h(r) r\|\nabla \varphi\|_{\infty} .
\end{aligned}
$$

Thus $(2 \pi)^{-1} f \in A^{h}(E)$, and so $\alpha(E) \leq C \gamma^{h}(E)$.

To deal with the capacity $\gamma_{+}^{h}$ it is useful to introduce the potential $U_{\mu}^{h}$ of a measure $\mu$. We consider the maximal operator $M^{h}$ :

$$
M^{h} \mu(x)=\sup _{r>0} \frac{\mu(B(x, r))}{h(r)} .
$$

Then we set

$$
U_{\mu}^{h}(x)=M^{h} \mu(x)+c_{\mu}(x) .
$$

Notice that, since $M^{h} \mu(x) \geq M \mu(x)$, we have $U_{\mu}^{h}(x) \geq U_{\mu}(x)$.

Lemma 4.2. For any compact set $E$, we have

$$
\gamma_{+}^{h}(E) \approx \sup \left\{\mu(E): \operatorname{supp}(\mu) \subset E, U_{\mu}^{h}(x) \leq 1 \forall x \in \mathbb{C}\right\},
$$

with absolute constants (independent of $h$ ). Also,

$$
\alpha_{+}(E) \approx \sup _{h} \gamma_{+}^{h}(E),
$$

with the supremum over all continuous functions $h:(0,+\infty) \longrightarrow(0,+\infty)$ satisfying (4.1) and (4.2), with $h(r) / r$ non decreasing.

Proof. First we will show that

$$
\gamma_{+}^{h}(E) \geq C^{-1} \sup \left\{\mu(E): \operatorname{supp}(\mu) \subset E, U_{\mu}^{h}(x) \leq 1 \forall x \in \mathbb{C}\right\} .
$$

Take $\mu$ supported on $E$ such that $U_{\mu}^{h}(x) \leq 1$ for all $x \in \mathbb{C}$. Since the Cauchy transform is bounded on $L^{2}(\mu)$ (with absolute constants), there exists some function $f$, with $0 \leq f \leq 1, \int f d \mu \geq \mu(E) / 2$, such that $\|\mathcal{C}(f d \mu)\|_{L^{\infty}(\mathbb{C})} \leq$ $C_{6}$. Then, $C^{-1} \mathcal{C}(f d \mu) \in A_{+}^{h}(E)$ for some constant $C$, since for any real function $\varphi \in \mathcal{C}^{\infty}$ supported on $B\left(x_{0}, r\right)$ we have

$$
\left|\int \mathcal{C}(f d \mu) \bar{\partial} \varphi d \mathcal{L}^{2}\right|=\left|\pi \int f \varphi d \mu\right| \leq \pi \mu\left(B\left(x_{0}, r\right)\right)\|\varphi\|_{\infty} \leq C h(r) r\|\nabla \varphi\|_{\infty} .
$$


To see the opposite inequality in (4.4), take $\mu$ supported on $E$ such that $\mu(E) \geq \gamma_{+}^{h}(E) / 2$, with $\mathcal{C} \mu \in A_{+}^{h}(E)$. Let $\varphi$ be a $\mathcal{C}^{\infty}$ function supported on $B\left(x_{0}, 2 r\right)$, with $\chi_{B\left(x_{0}, r\right)} \leq \varphi \leq 1$, and $\|\nabla \varphi\|_{\infty} \leq C r^{-1}$. Then,

$\mu\left(B\left(x_{0}, r\right)\right) \leq \int \varphi d \mu=\left|\pi^{-1} \int \mathcal{C} \mu \bar{\partial} \varphi d \mathcal{L}^{2}\right| \leq \pi^{-1} h(2 r) 2 r\|\nabla \varphi\|_{\infty} \leq C_{7} h(r)$.

Thus, $M^{h} \mu\left(x_{0}\right) \leq C_{7}$. Also, from (1.2) we deduce $c^{2}(\mu) \leq C_{8} \mu(E)$. Then, by Chebishev, there exists a compact set $F \subset E$ such that $\mu(F) \geq \mu(E) / 4$ and $c_{\mu \mid F}^{2}(x) \leq c_{\mu}^{2}(x) \leq 2 C_{8}$ for all $x \in F$. By the maximum principle (2.1), we get

$$
c_{\mu \mid F}^{2}(x) \leq C_{9} \quad \text { for all } x \in \mathbb{C} .
$$

Thus, $U_{\mu \mid F}^{h}(x) \leq C_{7}+C_{9}^{1 / 2}$ for all $x \in \mathbb{C}$. So some appropriate multiple of $\mu_{\mid F}$ fulfills the properties in the supremum on the right hand side of (4.4). This completes the proof of (4.4).

Let us turn our attention to (4.5) now. By Theorem 3.1 and (4.4) it is clear that

$$
\alpha_{+}(E) \geq C^{-1} \sup _{h} \gamma_{+}^{h}(E) .
$$

The opposite inequality follows like the proof of Lemma 4.1. We have to change $\alpha$ by $\alpha_{+}, \gamma^{h}$ by $\gamma_{+}^{h}, f$ by $\mathcal{C} \mu$ (where $\mu$ is some Radon measure supported on $E),\left|f^{\prime}(\infty)\right|$ by $\mu(E)$, etc.

We will show in Section 7 that $\gamma^{h} \approx \gamma_{+}^{h}$ with constants independent of $h$. Then, from the Lemmas 4.1 and 4.2 we will get

$$
\alpha(E) \leq C \sup _{h} \gamma^{h}(E) \approx \sup _{h} \gamma_{+}^{h}(E) \approx \alpha_{+}(E) .
$$

\section{Some PROperties of $\gamma^{h}$}

Lemma 5.1. (a) For any compact set $E \subset \mathbb{C}$ we have

$$
\gamma^{h}(E) \leq C_{10} h(\operatorname{diam}(E)) .
$$

(b) For any closed disk $D_{r}$ of radius $r$ we have

$$
\gamma^{h}\left(D_{r}\right) \approx \gamma_{+}^{h}\left(D_{r}\right) \approx h(r) .
$$

(c) If $R$ is a rectangle with side lengths $L$ and $\ell$, with $L \geq \ell$, then

$$
\gamma^{h}(R) \approx \gamma_{+}^{h}(R) \approx L \frac{h(\ell)}{\ell} .
$$

Proof. (a) Suppose that $E \subset B\left(x_{0}, r\right)$. Take a function $\varphi \in \mathcal{C}^{\infty}$ supported on $B\left(x_{0}, 2 r\right)$, with $\chi_{B\left(x_{0}, r\right)} \leq \varphi \leq \chi_{B\left(x_{0}, 2 r\right)}$, and $\|\nabla \varphi\|_{\infty} \leq$ $C r^{-1}$. Then, if $f \in A^{h}(E)$,

$$
\left|f^{\prime}(\infty)\right|=\left|C \int f \bar{\partial} \varphi d \mathcal{L}^{2}\right| \leq C h(2 r) r\|\nabla \varphi\|_{\infty} \leq C h(r) .
$$


(b) We only have to show that $\gamma_{+}^{h}\left(D_{r}\right) \geq C^{-1} h(r)$. Consider the measure

$$
d \mu=\frac{h(r)}{r^{2}} d \mathcal{L}_{\mid D_{r}}^{2}
$$

Then, for all $x \in \mathbb{C}$ and $0 \leq s \leq r$,

$$
\mu(B(x, s)) \leq \frac{s^{2}}{r^{2}} h(r) \leq C h(s)
$$

The last inequality follows from (4.3). In the case $s>r$, we have

$$
\mu(B(x, s)) \leq \mu\left(D_{r}\right)=\pi h(r) \leq \pi h(s) .
$$

It is also straightforward to check that $\mathcal{C} \mu$ is a bounded function (with absolute constants). So there exists some constant $C$ such that $C^{-1} \mathcal{C}_{\mu} \in A_{+}^{h}\left(D_{r}\right)$, and then $\gamma_{+}^{h}\left(D_{r}\right) \geq C^{-1} \mu\left(D_{r}\right)=C^{-1} h(r)$.

(c) This statement will be proved after Lemma 5.2 below.

Notice that, unlike for the usual analytic capacity $\gamma$, in general, for $\lambda>0$,

$$
\gamma^{h}(\lambda E) \neq \lambda \gamma^{h}(E)
$$

In next lemma we show that Vitushkin's localization operator $V_{\varphi}$, defined by

$$
V_{\varphi} f:=\frac{1}{\pi z} *(\varphi \bar{\partial} f)=\varphi f-\frac{1}{\pi z} *(f \bar{\partial} \varphi)
$$

where $\varphi$ is a compactly supported $\mathcal{C}^{\infty}$ function, behaves well with respect to $\gamma^{h}$.

Lemma 5.2. Let $E \subset \mathbb{C}$ be compact and $f \in A^{h}(E)$. Let $\varphi$ be a $\mathcal{C}^{\infty}$ function supported on $\bar{B}\left(x_{0}, r\right)$, such that $\|\varphi\|_{\infty} \leq C_{11}$ and $\|\nabla \varphi\|_{\infty} \leq C_{11} r^{-1}$. Then there exists some constant $C$ depending on $C_{11}$ such that $C^{-1} V_{\varphi} f \in A^{h}(E \cap$ $\left.\bar{B}\left(x_{0}, r\right)\right)$.

Proof. It is well known that if $f \in A^{h}(E)$, then $V_{\varphi} f$ is a bounded function analytic in $\mathbb{C} \backslash\left(E \cap \bar{B}\left(x_{0}, r\right)\right)$. So we only have to check that

$$
\left|\int V_{\varphi} f \bar{\partial} \psi d \mathcal{L}^{2}\right| \leq C h(s) s\|\nabla \psi\|_{\infty}
$$

for any $\mathcal{C}^{\infty}$ function $\psi$ supported on a ball $B\left(y_{0}, s\right)$. We denote by $\langle\cdot, \cdot\rangle$ the pairing between the space of distributions $\mathcal{D}^{\prime}$ and $\mathcal{D}:=\mathcal{C}_{c}^{\infty}\left(\mathbb{R}^{n}\right)$. We have

$$
\int V_{\varphi} f \bar{\partial} \psi d \mathcal{L}^{2}=-\left\langle\bar{\partial}\left(V_{\varphi} f\right), \psi\right\rangle=-\langle\bar{\partial} f, \varphi \psi\rangle=\int f \bar{\partial}(\varphi \psi) d \mathcal{L}^{2}
$$

Thus, if we denote $d=\operatorname{diam}(\operatorname{supp}(\varphi \psi))$, we get

$$
\left|\int V_{\varphi} f \bar{\partial} \psi d \mathcal{L}^{2}\right| \leq C h(d) d\|\nabla(\varphi \psi)\|_{\infty}
$$


We have

$$
\begin{aligned}
\|\nabla(\varphi \psi)\|_{\infty} & \leq\|\varphi\|_{\infty}\|\nabla \psi\|_{\infty}+\|\psi\|_{\infty}\|\nabla \varphi\|_{\infty} \\
& \leq C\|\nabla \psi\|_{\infty}+C\left(\|\nabla \psi\|_{\infty} s\right) \frac{1}{r} \leq C\left(1+\frac{s}{r}\right)\|\nabla \psi\|_{\infty} .
\end{aligned}
$$

Notice that $d \leq \min (2 s, 2 r)$, and since $h$ is non decreasing, we get $h(d) \leq$ $h(2 s) \leq 4 h(s)$, and then

$$
\left|\int V_{\varphi} f \bar{\partial} \psi d \mathcal{L}^{2}\right| \leq C\left(h(d) d+h(d) \frac{d s}{r}\right)\|\nabla \psi\|_{\infty} \leq C h(s) s .
$$

Proof of Lemma 5.1 (c). Given a rectangle $R$ with side lengths $L$ and $\ell$, with $L \geq \ell$, we have to show that

$$
\gamma^{h}(R) \approx \gamma_{+}^{h}(R) \approx L \frac{h(\ell)}{\ell} .
$$

If $R$ is a square, then from (b) in Lemma 5.1, it easily follows that $\gamma^{h}(R) \approx$ $h(\ell)$, and we are done.

Suppose now that $L=N \ell$, where $N$ is some positive integer. So $R=$ $\bigcup_{i=1}^{N} Q_{i}$, where each $Q_{i}$ is a square of side length $\ell$, and different squares $Q_{i}$ have disjoint interiors. Let $\varphi_{i}, 1 \leq i \leq N$, be non negative $\mathcal{C}^{\infty}$ functions such that, for each $i, \operatorname{supp}\left(\varphi_{i}\right) \subset 2 Q_{i},\left\|\nabla \varphi_{i}\right\|_{\infty} \leq C \ell^{-1}$, and $\sum_{i=1}^{N} \varphi_{i}=1$ in a neighborhood of $R$. If $f \in A^{h}(R)$, then $C^{-1} V_{\varphi_{i}} f \in A^{h}\left(2 Q_{i}\right)$ and so

$$
\left|\left(V_{\varphi_{i}} f\right)^{\prime}(\infty)\right| \leq C \gamma^{h}\left(2 Q_{i}\right) \approx h(\ell)
$$

Thus,

$$
\left|f^{\prime}(\infty)\right| \leq \sum_{i=1}^{N}\left|\left(V_{\varphi_{i}} f\right)^{\prime}(\infty)\right| \leq C N h(\ell),
$$

which yields $\gamma^{h}(R) \leq C N h(\ell)$.

For a general rectangle $R$, let $N$ be the least integer such that $N \geq L / \ell$. Let $R_{0}$ be a rectangle containing $R$ with side lengths $N \ell$ and $\ell$. Then,

$$
\gamma^{h}(R) \leq \gamma^{h}\left(R_{0}\right) \leq C N h(\ell) \leq C L \frac{h(\ell)}{\ell} .
$$

Let us see now that $\gamma_{+}^{h}(R) \geq C^{-1} L h(\ell) / \ell$. We will use (4.4). Consider the measure

$$
\mu=\frac{h(\ell)}{\ell^{2}} \mathcal{L}_{\mid R}^{2}
$$

Then $\mu(R)=L h(\ell) / \ell$, and also it is easy to check that $c_{\mu}(x) \leq C$ for all $x \in \mathbb{C}$. Further, for any ball $B(x, r)$ we have

$$
\mu(B(x, r))=\frac{h(\ell)}{\ell^{2}} \mathcal{L}^{2}(B(x, r) \cap R) \leq \frac{C h(\ell)}{\ell^{2}} \min \left(r^{2}, r \ell\right) .
$$

Thus, if $r \geq \ell$,

$$
\mu(B(x, r)) \leq \frac{C h(\ell) r}{\ell} \leq \frac{C h(r) r}{r}=C h(r),
$$


since $h(t) / t$ is a non decreasing function of $t$. If $r<\ell$, we get

$$
\mu(B(x, r)) \leq \frac{C h(\ell) r^{2}}{\ell^{2}} \leq C h(r),
$$

by (4.3). So $M^{h} \mu(x) \leq C$ for all $x \in \mathbb{C}$. Therefore,

$$
\gamma_{+}^{h}(R) \geq C^{-1} \mu(R)=C^{-1} \operatorname{Lh}(\ell) / \ell .
$$

Using Lemma 5.2, we can prove the semiaddivity of $\gamma^{h}$ in some special cases:

Lemma 5.3. (a) For a compact set $E \subset \mathbb{C}$ and a closed disk $D \subset \mathbb{C}$, we have

$$
\gamma^{h}(E \cup D) \leq C\left(\gamma^{h}(E)+\gamma^{h}(D)\right)
$$

(b) Given two rectangles $R, T \subset \mathbb{C}$ (we do not assume their sides to be parallel to the axes),

$$
\gamma^{h}(R \cup T) \leq C\left(\gamma^{h}(R)+\gamma^{h}(T)\right) .
$$

Proof. Take $f \in A^{h}(E \cup D)$ such that $\gamma^{h}(E \cup D) \leq 2\left|f^{\prime}(\infty)\right|$. Let $\varphi \in \mathcal{C}^{\infty}$ be supported on $2 D$ such that $\varphi=1$ in a neighborhood of $D$, with $\|\nabla \varphi\|_{\infty} \leq$ $C r^{-1}$, where $r$ is the radius of $D$. Now set $f_{1}=V_{\varphi} f$ and $f_{2}=f-f_{1}$. By Lemma 5.2, $C^{-1} f_{1} \in A^{h}(2 D)$ for some constant $C$. So we deduce that $C^{-1} f_{2} \in A^{h}(E)$ for another constant $C$. Therefore,

$$
\gamma^{h}(E \cup D) \leq 2\left(\left|f_{1}^{\prime}(\infty)\right|+\left|f_{2}^{\prime}(\infty)\right|\right) \leq C\left(\gamma^{h}(2 D)+\gamma^{h}(E)\right) .
$$

Then (a) follows from the fact that $\gamma^{h}(2 D) \approx h(2 r) \approx h(r) \approx \gamma^{h}(D)$.

Consider now the rectangles $R$ and $T$. Let $L_{R}, \ell_{R}$ be the side lengths of $R$, and $L_{T}, \ell_{T}$ the side lengths of $T$. Suppose $\ell_{R} \leq L_{R}$ and $\ell_{T} \leq L_{T}$. Assume first that $L_{R}=M \ell_{R}$ and $L_{T}=N \ell_{T}$ with $M, N \in \mathbb{Z}$. So we can write $R=\bigcup_{i=1}^{M} P_{i}$ and $T=\bigcup_{j=1}^{N} Q_{j}$, where $P_{i}$ and $Q_{j}$ are squares of side lengths $\ell_{R}$ and $\ell_{T}$ respectively. Take positive functions $\varphi_{i} \in \mathcal{C}^{\infty}, 1 \leq i \leq M$, with $\operatorname{supp}\left(\varphi_{i}\right) \subset 2 P_{i}$ for each $i$, satisfying $\sum_{i=1}^{M} \varphi_{i} \leq 1$ on $\mathbb{C}, \sum_{i=1}^{M} \varphi_{i}=1$ on $R$, and $\left\|\nabla \varphi_{i}\right\|_{\infty} \leq C \ell_{R}^{-1}$. Let $\psi_{j}, 1 \leq j \leq N$ be the analogous functions for $T$. Suppose that $\ell_{R} \geq \ell_{T}$. Set $\widetilde{\psi}_{j}=\psi_{j}\left(1-\sum_{i=1}^{M} \varphi_{i}\right)$. Then,

$$
\sum_{i=1}^{M} \varphi_{i}+\sum_{j=1}^{N} \widetilde{\psi}_{j}=1
$$

on $R \cup T$ and so, given $f \in A^{h}(R \cup T)$, we have

$$
f=\sum_{i=1}^{M} V_{\varphi_{i}} f+\sum_{j=1}^{N} V_{\widetilde{\psi}_{j}} f .
$$


Moreover, for some $C>0, C V_{\varphi_{i}} f \in A^{h}\left(2 P_{i}\right)$, and also $C V_{\widetilde{\psi}_{j}} f \in A^{h}\left(2 Q_{j}\right)$ (because $\left\|\widetilde{\psi}_{j}\right\|_{\infty} \leq 1$ and $\left\|\nabla \widetilde{\psi}_{j}\right\|_{\infty} \leq C \ell_{T}^{-1}$ ). Thus,

$$
\begin{aligned}
\left|f^{\prime}(\infty)\right| & \leq \sum_{i=1}^{M} \gamma^{h}\left(2 P_{i}\right)+\sum_{j=1}^{N} \gamma^{h}\left(2 Q_{j}\right) \\
& \leq C\left(M h\left(\ell_{R}\right)+N h\left(\ell_{T}\right)\right) \leq C\left(\gamma^{h}(R)+\gamma^{h}(T)\right) .
\end{aligned}
$$

Suppose now that $R$ and $T$ are arbitrary rectangles. Let $M$ be the least integer such that $M \geq L_{R} / \ell_{R}$, and $N$ the least integer such that $N \geq L_{T} / \ell_{T}$. Let $R_{0}$ be a rectangle containing $R$ with side lengths $\ell_{R}$ and $M \ell_{R}$, and $T_{0}$ another rectangle containing $T$ with side lengths $\ell_{T}$ and $N \ell_{T}$. Then,

$$
\gamma^{h}(R \cup T) \leq \gamma^{h}\left(R_{0} \cup T_{0}\right) \leq C\left(\gamma^{h}\left(R_{0}\right)+\gamma^{h}\left(T_{0}\right)\right) \leq C\left(\gamma^{h}(R)+\gamma^{h}(T)\right) .
$$

Another property of $\gamma^{h}$ is the following.

Lemma 5.4. Suppose that $E \subset B\left(x_{0}, \delta\right)$. Let $f \in A^{h}(E)$ and consider the Laurent expansion of $f$ centered at $x_{0}$ :

$$
f(z)=\sum_{n=1}^{\infty} \frac{a_{n}}{\left(z-x_{0}\right)^{n}}, \quad\left|z-x_{0}\right|>\delta .
$$

We have $\left|a_{n}\right| \leq C n\left(\frac{3}{2} \delta\right)^{n-1} \gamma^{h}(E)$.

Proof. We argue as in [Ve1, p.444]. Suppose for simplicity that $x_{0}=0$. The function

$$
g(z)=z^{n-1}\left(f(z)-\sum_{j=1}^{n-1} \frac{a_{j}}{z^{j}}\right)
$$

is analytic outside $E$ and satisfies $g(\infty)=0$. We are going to show that $g /\left(C n\left(\frac{3}{2} \delta\right)^{n-1}\right) \in A^{h}(E)$ for some constant $C$. Observe that this fact implies that

$$
\left|a_{n}\right|=\left|g^{\prime}(\infty)\right| \leq C n\left(\frac{3}{2} \delta\right)^{n-1} \gamma^{h}(E)
$$

To show that $g /\left(C n \frac{3}{2} \delta^{n-1}\right) \in A^{h}(E)$, we intend to apply Lemma 5.2. Notice that

$$
\bar{\partial} g=z^{n-1} \bar{\partial} f=z^{n-1} \rho \bar{\partial} f,
$$

where $\rho$ is a $\mathcal{C}^{\infty}$ function supported on $B\left(0, \frac{3}{2} \delta\right)$, identically 1 on $B(0, \delta)$, with $\|\rho\|_{\infty} \leq 1$ and $\|\nabla \rho\|_{\infty} \leq C / \delta$. We denote $\varphi(z):=\frac{1}{n\left(\frac{3}{2} \delta\right)^{n-1}} z^{n-1} \rho(z)$. From (5.2), it follows that

$$
\frac{1}{n\left(\frac{3}{2} \delta\right)^{n-1}} g=V_{\varphi} f
$$


We have $\operatorname{supp}(\varphi) \subset B\left(0, \frac{3}{2} \delta\right)$, and also

$$
\|\varphi\|_{\infty}=\frac{1}{n\left(\frac{3}{2} \delta\right)^{n-1}}\left\|z^{n-1} \rho\right\|_{\infty} \leq \frac{1}{n} \leq 1 .
$$

Now it only remains to estimate $\|\nabla \varphi\|_{\infty}$ :

$$
\begin{aligned}
\|\nabla \varphi\|_{\infty} & \leq \frac{1}{n\left(\frac{3}{2} \delta\right)^{n-1}}\left(\left\|\nabla\left(z^{n-1}\right)\right\|_{\infty, B\left(0, \frac{3}{2} \delta\right)}\|\rho\|_{\infty}+\left\|z^{n-1}\right\|_{\infty, B\left(0, \frac{3}{2} \delta\right)}\|\nabla \rho\|_{\infty}\right) \\
& \leq \frac{1}{n\left(\frac{3}{2} \delta\right)^{n-1}} C n\left(\frac{3}{2} \delta\right)^{n-2}=\frac{C}{\delta} .
\end{aligned}
$$

Hence by Lemma 5.2 we are done.

From the preceding lemma we deduce the following property of $\gamma^{h}$, which will be very useful in Section 7.

Lemma 5.5. Suppose that $f \in A^{h}(E)$ satisfies $f^{\prime}(\infty)=0$. Then, for all $z \in \mathbb{C}$ such that $\operatorname{dist}(z, E) \geq 2 \operatorname{diam}(E)$, we have

$$
|f(z)| \leq C \frac{\operatorname{diam}(E) \gamma^{h}(E)}{\operatorname{dist}(z, E)^{2}} .
$$

Proof. Set $\delta=\operatorname{diam}(E)$. Take $x_{0} \in E$. Then, $E \subset B\left(x_{0}, \delta\right)$. We consider the Laurent expansion of $f$ centered at $x_{0}$ :

$$
f(z)=\sum_{n=2}^{\infty} \frac{a_{n}}{\left(z-x_{0}\right)^{n}}, \quad\left|z-x_{0}\right|>\delta .
$$

Notice that if $\operatorname{dist}(z, E) \geq 2 \operatorname{diam}(E)$, then $\left|z-x_{0}\right| \geq 2 \delta$ and, from the preceding lemma, we deduce

$$
|f(z)| \leq \sum_{n=2}^{\infty} \frac{C n\left(\frac{3}{2} \delta\right)^{n-1} \gamma^{h}(E)}{\left|z-x_{0}\right|^{n}} \leq \frac{C \delta \gamma^{h}(E)}{\left|z-x_{0}\right|^{2}}
$$

\section{Some PROperties of $\gamma_{+}^{h}$}

We denote

$$
\Sigma^{h}(E)=\left\{\mu \in M_{+}(\mathbb{C}): \operatorname{supp}(\mu) \subset E, M^{h} \mu(x) \leq 1 \forall x \in \mathbb{C}\right\} .
$$

Lemma 6.1. The following properties of $\gamma_{+}^{h}$ hold:

(a) For any compact set $E \subset \mathbb{C}$,

$$
\gamma_{+}^{h}(E) \approx \sup _{\mu \in \Sigma^{h}(E)} \frac{\mu(E)^{2}}{\mu(E)+c^{2}(\mu)} .
$$

(b) For any compact set $E \subset \mathbb{C}$,

$$
\gamma_{+}^{h}(E) \leq C \mathcal{H}_{\infty}^{h}(E) .
$$


(c) If $E, F \subset \mathbb{C}$ are compact, then

$$
\gamma_{+}^{h}(E \cup F) \leq C\left(\gamma_{+}^{h}(E)+\gamma_{+}^{h}(F)\right) .
$$

(d) For any finite Radon measure $\mu$ on $\mathbb{C}$ and any $\lambda>0$,

$$
\gamma_{+}^{h}\left\{x \in \mathbb{C}: U_{\mu}^{h}(x)>\lambda\right\} \leq \frac{C \mu(\mathbb{C})}{\lambda} .
$$

(e) Let $\left\{E_{n}\right\}_{n \geq 1}$ be a sequence of compact sets in $\mathbb{C}$ such that $E_{n+1} \subset E_{n}$ for all $n$. Set $E=\bigcap_{n} E_{n}$. Then, $\gamma^{h}(E)=\lim _{n \rightarrow \infty} \gamma^{h}\left(E_{n}\right)$.

In (b), $\mathcal{H}_{\infty}^{h}$ stands for the $h$-Hausdorff content. We recall that $\mathcal{H}_{\infty}^{h}$ fulfills the following weak $(1,1)$ inequality:

$$
\mathcal{H}_{\infty}^{h}\left\{x \in \mathbb{C}: M^{h} \mu(x)>\lambda\right\} \leq \frac{C \mu(\mathbb{C})}{\lambda},
$$

for any Radon measure $\mu$ and all $\lambda>0$. This can be proved by means of the $5 r$ covering theorem of Vitali (using the doubling property of $h$ ). Notice also that the capacitary version (for $\gamma_{+}^{h}$ ) of (6.1) is (d) in the lemma above.

Proof of Lemma 6.1. (a) follows from the characterization (4.4) of $\gamma_{+}^{h}$. The arguments are analogous to the ones for $\gamma_{+}$in [To2, Theorem 5.1].

The proof of (b) is also derived easily from (4.4), and is left for the reader again.

Also, (c) is another straightforward consequence of (4.4).

The property (d) is proved as the analogous inequality for $\gamma_{+}$in [To4, Theorem 3.1]. One has to interchange $\gamma_{+}$by $\gamma_{+}^{h}$, the maximal operator $M$ by $M^{h}$, etc. Notice by the way that the maximum principle (2.1) (which is needed in the proof of [To4, Theorem 3.1]) also holds with $M^{h}$ instead of $M$, since $M \mu \leq M^{h} \mu$ for any measure $\mu$.

The proof of (e) follows by standard arguments and is left for the reader.

The capacities $\gamma_{+}^{h}$ also satisfy the following property.

Lemma 6.2. For $E \subset \mathbb{C}$ compact, we have

$$
\gamma_{+}^{h}(E) \approx \inf \left\{\mu(\mathbb{C}): \mu \in M_{+}(\mathbb{C}), U_{\mu}^{h}(x) \geq 1 \forall x \in E\right\} .
$$

The constants involved in (6.2) do not depend on $h$. The lemma is proved by arguments analogous to the ones of [To4, Theorem 3.3]. It follows from (c) in Lemma 6.1 and next lemma applied to a suitable discrete version $\widetilde{E}$ of $E$.

Lemma 6.3. Consider a grid of squares of side length $\delta>0$ in $\mathbb{C}$ with sides parallel to the axes. Take a finite collection of closed squares $\left\{Q_{i}\right\}_{i \in I}$ of the grid. For each $i \in I$, let $P_{i}$ be the closed square of side length $\delta / 4$ concentric with $Q_{i}$ (and sides parallel to the axes). Set $\widetilde{E}=\bigcup_{i \in I} Q_{i}$. Let $\Sigma_{0}^{h}(\widetilde{E})$ be the 
subset of $\Sigma_{h}(\widetilde{E})$ of measures $\mu$ of the form $\mu=\sum_{i \in I} a_{i} \mathcal{L}_{\mid P_{i}}^{2}, a_{i} \geq 0$. Then there exists a measure $\sigma \in \Sigma_{0}^{h}(\widetilde{E})$ such that

$$
\frac{\|\sigma\|^{2}}{\|\sigma\|+c^{2}(\sigma)}=\sup _{\mu \in \Sigma_{0}^{h}(E)} \frac{\|\mu\|^{2}}{\|\mu\|+c^{2}(\mu)} .
$$

The maximal measure $\sigma$ satisfies $c^{2}(\sigma) \leq 2\|\sigma\|$ and $U_{\sigma}^{h}(x) \geq C_{12}$ for all $x \in \widetilde{E}$, where $C_{12}$ is some absolute constant.

This lemma follows from a variational argument analogous to the one of [To4, Lemma 3.6]. The details of the proofs of this lemma and the preceding one are left for the reader again.

\section{Comparability Between $\gamma^{h}$ And $\gamma_{+}^{h}$}

In this section we will prove the following result. This will be the last step of the proof of Theorem 1.4.

Theorem 7.1. There exists an absolute constant $C$ (not depending on h) such that

$$
\gamma^{h}(E) \leq C \gamma_{+}^{h}(E)
$$

for any compact set $E \subset \mathbb{C}$.

The proof of this theorem is quite similar to the proof of the comparability between $\gamma$ and $\gamma_{+}$in [To5, Theorem 1.1]. We must show that there exists some measure $\mu$ supported on $E$, with $\mu(E) \approx \gamma^{h}(E)$, such that $\mu(B(x, r)) \leq$ $h(r)$, and with the Cauchy transform is bounded on $L^{2}(\mu)$ with absolute constants. In order to construct $\mu$, we will need to apply an induction argument: We will prove that $\gamma^{h}(E \cap R) \approx \gamma_{+}^{h}(E \cap R)$ for all the rectangles $R$, by induction on the size of $R$.

7.1. The First Main lemma. Next lemma, which is one of the main steps for the proof of Theorem 7.1, deals with the construction of an intermediate set $F$ and some measures $\mu$ (non negative) and $\nu$ (complex) suitable for the application of a $T b$ theorem. Unlike in [To5, Lemma 5.1], we do not assume $E$ to be made up a finite union of segments, or squares, or rectangles, etc. Now $E$ is supposed to be an arbitrary compact set.

Lemma 7.2 (First MainLemma). Suppose that $\gamma_{+}^{h}(E) \leq C_{13} h(\operatorname{diam}(E))$, with $C_{13}>0$ small enough. Then there exists a compact set $F=\bigcup_{i \in I} Q_{i}$, with $\sum_{i \in I} \chi_{10 Q_{i}} \leq C$, such that

(a) $E \subset F$ and $\gamma_{+}^{h}(F) \leq C \gamma_{+}^{h}(E)$,

(b) $\sum_{i \in I} \gamma_{+}^{h}\left(E \cap 2 Q_{i}\right) \leq C \gamma_{+}^{h}(E)$,

(c) $\operatorname{diam}\left(Q_{i}\right) \leq \frac{1}{10} \operatorname{diam}(E)$ for every $i \in I$.

Let $A \geq 1$ be some fixed constant. Suppose that $\gamma^{h}\left(E \cap 2 Q_{i}\right) \leq A \gamma_{+}^{h}\left(E \cap 2 Q_{i}\right)$ for all $i \in I$. If $\gamma^{h}(E) \geq A \gamma_{+}^{h}(E)$, then there exist a positive Radon measure $\mu$ and a complex Radon measure $\nu$, both supported on $F$, and a subset $H \subset$ $F$, such that 
(d) $C_{a}^{-1} \gamma^{h}(E) \leq \mu(F) \leq C_{a} \gamma^{h}(E)$,

(e) $d \nu=b d \mu$, with $\|b\|_{L^{\infty}(\mu)} \leq C_{b}$,

(f) $|\nu(F)| \geq \gamma^{h}(E) / 2$,

(g) $\int_{F \backslash H} \mathcal{C}_{*} \nu d \mu \leq C_{c} \mu(F)$,

(h) $\left|\int \varphi d \nu\right| \leq C h(r) r\|\nabla \varphi\|_{\infty}$ for any function $\varphi \in \mathcal{C}^{\infty}$ supported on a ball of radius $r$.

(i) If $\mu(B(x, r))>C_{h} h(r)$ (for some big constant $\left.C_{h}\right)$, then $B(x, r) \cap$ $F \subset H$. In particular, $\mu(B(x, r)) \leq C_{h} h(r)$ for all $x \in F \backslash H, r>0$.

(j) $H$ is of the form $H=\bigcup_{k \in I_{H}} B\left(x_{k}, r_{k}\right) \cap F$, with $\sum_{k \in I_{H}} h\left(r_{k}\right) \leq$ $\varepsilon \mu(F)$, for $0<\varepsilon<1 / 10$ arbitrarily small (choosing $C_{h}$ big enough).

The constants $C_{13}, C, C_{a}, C_{b}, C_{c}, C_{h}, \varepsilon, \delta$ do not depend on $A$. They are absolute constants.

Let us insist on the fact that all the constants different from $A$ which appear in the lemma do not depend on $A$. This will be essential for the proof of Theorem 7.1. We have preferred to use the notation $C_{a}, C_{b}, C_{c}, C_{h}$ instead of $C_{14}, \ldots, C_{17}$, say, because these constants will play an important role in the proof of Theorem 7.1. Of course, the constants $C_{b}$ and $C_{h}$ do not depend either on $b$ or $h$ (they are absolute constants).

The set $F$ has to be understood as an approximation of $E$ at an intermediate scale. The first part of the lemma, which deals with the construction of $F$ and the properties (a)-(c), is proved in Subsections 7.2-7.4. The choice of the squares $Q_{i}$ which satisfy (a) and (b) is one of the key steps of the proof Theorem 7.1. Notice that (a) implies that the squares $Q_{i}$ are not too big and (b) that they are not too small. That is, they belong to some intermediate scale. The property (b) will be essential for the proof of (d). On the other hand, the assertion (c) will only be used in the final induction argument, in Subsection 7.14.

The properties (d), (e), (f) and (g) are proved in Subsections 7.5 and 7.7. These are the basic properties which must satisfy $\mu$ and $\nu$ in order to apply a $T(b)$ theorem with absolute constants. To prove (d) we will need the assumptions in the paragraph after (c) in the lemma. In (g) notice that instead of the $L^{\infty}(\mu)$ or $B M O(\mu)$ norm of $\mathcal{C} \nu$, we estimate the $L^{1}(\mu)$ norm of $\mathcal{C}_{*} \nu$ outside $H$. The statement in (h) prevents $\nu$ from being too much concentrated, in a sense. It is a smooth version of the inequality $|\nu(B(x, r))| \leq C h(r)$, which we don't know if it holds.

Roughly speaking, the exceptional set $H$ contains the part of $\mu$ where the measure $\mu$ is too much concentrated. The properties (i) and (j) describe $H$ and are proved in Subsection 7.6. Observe that $(\mathrm{j})$ means that $H$ is a rather small set, in a sense.

7.2. The construction of $F$ and the proof of (a) in First Main Lemma. Let $\sigma \in M_{+}(\mathbb{C})$ be a measure satisfying $\sigma(E) \approx \gamma_{+}^{h}(E)$ and $U_{\sigma}^{h}(x) \geq 1$ for all $x \in E$ (recall Lemma 6.2). Let $\lambda$ be some constant 
with $0<\lambda \leq 10^{-8}$ which will be fixed below. Let $\Omega_{\lambda} \subset \mathbb{C}$ be the open set

$$
\Omega_{\lambda}:=\left\{x \in \mathbb{C}: U_{\sigma}^{h}(x)>\lambda\right\} .
$$

Notice that $E \subset \Omega_{\lambda}$, and by (d) in Lemma 6.1 we have

$$
\gamma_{+}^{h}\left(\Omega_{\lambda}\right) \leq C \lambda^{-1} \sigma(E) \leq C \lambda^{-1} \gamma_{+}^{h}(E) .
$$

Let $\Omega_{\lambda}=\bigcup_{i \in J} Q_{i}$ be a Whitney decomposition of $\Omega_{\lambda}$, where $\left\{Q_{i}\right\}_{i \in J}$ is the usual family of Whitney squares with disjoint interiors, satisfying $20 Q_{i} \subset \Omega_{\lambda}, R Q_{i} \cap\left(\mathbb{C} \backslash \Omega_{\lambda}\right) \neq \varnothing$ (where $R>20$ is some fixed absolute constant), and $\sum_{i \in J} \chi_{10 Q_{i}} \leq C$.

Let $\left\{Q_{i}\right\}_{i \in I}, I \subset J$, be the subfamily of squares such that $2 Q_{i} \cap E \neq \varnothing$. We set

$$
F:=\bigcup_{i \in I} Q_{i}
$$

Observe that the property (a) of First Main Lemma is a consequence of (7.1).

On the other hand, since $E$ is compact, it is easy to check that the collection of squares $Q_{i}, i \in I$, is finite, and as a consequence, $F$ is also compact.

7.3. Proof of (b) in First Main Lemma. Let us see now that (b) holds if $\lambda$ has been chosen small enough. Arguing as in [To5, Subsection 5.2], one can check that if $x \in E \cap 2 Q_{i}$ for some $i \in I$, then

$$
U_{\sigma \mid 4 Q_{i}}^{h}(x)>1 / 4,
$$

assuming that $\lambda$ is small enough. This implies $E \cap 2 Q_{i} \subset\left\{U_{\sigma \mid 4 Q_{i}}^{h}>1 / 4\right\}$ and then, by (d) in Lemma 6.1, we have

$$
\gamma_{+}^{h}\left(E \cap 2 Q_{i}\right) \leq C \sigma\left(4 Q_{i}\right) .
$$

Using the finite overlap of the squares $4 Q_{i}$, we deduce

$$
\sum_{i \in I} \gamma_{+}^{h}\left(E \cap 2 Q_{i}\right) \leq C \sum_{i \in I} \sigma\left(4 Q_{i}\right) \leq C \sigma(E) \leq C \gamma_{+}^{h}(E)
$$

7.4. Proof of (c) in First Main Lemma. Now we have to show that

$$
\operatorname{diam}\left(Q_{i}\right) \leq \frac{1}{10} \operatorname{diam}(E) .
$$

It is immediate to check that

$$
U_{\sigma}^{h}(x) \leq \frac{10 \sigma(E)}{h(\operatorname{dist}(x, E))}
$$

for all $x \notin E$. Thus, for $x \in \Omega_{\lambda} \backslash E$ we have

$$
\lambda<U_{\sigma}^{h}(x) \leq \frac{10 \sigma(E)}{h(\operatorname{dist}(x, E))} .
$$


Therefore,

$$
\begin{aligned}
h(\operatorname{dist}(x, E)) & \leq 10 \lambda^{-1} \sigma(E) \leq C \lambda^{-1} \gamma_{+}^{h}(E) \\
& \leq \frac{1}{401} h(\operatorname{diam}(E))<h\left(\frac{\operatorname{diam}(E)}{10}\right),
\end{aligned}
$$

assuming $C_{13}$ in First Main Lemma small enough for the third inequality, and using (4.3) in the last one. Since $h$ in non decreasing, we deduce $\operatorname{dist}(x, E)<\operatorname{diam}(E) / 10$.

As a consequence, $\operatorname{diam}\left(\Omega_{\lambda}\right) \leq \frac{6}{5} \operatorname{diam}(E)$. Since $20 Q_{i} \subset \Omega_{\lambda}$ for each $i \in I$, we have

$$
20 \operatorname{diam}\left(Q_{i}\right) \leq \operatorname{diam}\left(\Omega_{\lambda}\right) \leq \frac{6}{5} \operatorname{diam}(E),
$$

which implies (7.3).

7.5. The construction of $\mu$ and $\nu$ and the proof of (d)-(f) in First Main Lemma. It is easily seen that there exists a family of $\mathcal{C}^{\infty}$ functions $\left\{g_{i}\right\}_{i \in J}$ such that, for each $i \in J, \operatorname{supp}\left(g_{i}\right) \subset 2 Q_{i}, 0 \leq g_{i} \leq 1$, and $\left\|\nabla g_{i}\right\|_{\infty} \leq$ $C / \ell\left(Q_{i}\right)$, so that $\sum_{i \in J} g_{i}=1$ on $\Omega_{\lambda}$. Notice that by the definition of $I$ in Subsection 7.2 , we also have $\sum_{i \in I} g_{i}=1$ on $E$.

Let $f \in A^{h}(E)$ be such that $f^{\prime}(\infty) \geq \gamma^{h}(E) / 2$. In a sense, the measure $\nu$ will be a suitable approximation of the distribution $\pi^{-1} \bar{\partial} f$ [remember that $\left.f=\mathcal{C}\left(\pi^{-1} \bar{\partial} f\right)\right]$.

First we introduce the measure $\mu$. For each $i \in I$, let $\Delta_{i}$ be a disk concentric with $Q_{i}$, of radius $r_{i}$ such that $h\left(r_{i}\right)=\gamma^{h}\left(E \cap 2 Q_{i}\right) /\left(10^{3} C_{10}\right)$ (this radius exists by continuity). Using Lemma 5.1 (a), it is easy to check that $\Delta_{i} \subset \frac{1}{2} Q_{i}$ for each $i$. We set

$$
\mu=\sum_{i \in I} \frac{h\left(r_{i}\right)}{\mathcal{L}^{2}\left(\Delta_{i}\right)} \mathcal{L}^{2} \mid \Delta_{i}
$$

Observe that

$$
\gamma^{h}\left(\Delta_{i}\right) \approx h\left(r_{i}\right)=\frac{1}{10^{3} C_{10}} \gamma^{h}\left(E \cap 2 Q_{i}\right)=\mu\left(\Delta_{i}\right) .
$$

Let us define $\nu$ now:

$$
\nu=\sum_{i \in I} \frac{1}{\mathcal{L}^{2}\left(\Delta_{i}\right)}\left\langle\pi^{-1} \bar{\partial} f, g_{i}\right\rangle \cdot \mathcal{L}^{2}\left|\Delta_{i}=\sum_{i \in I} \frac{-1}{\pi \mathcal{L}^{2}\left(\Delta_{i}\right)} \int f \bar{\partial} g_{i} d \mathcal{L}^{2} \cdot \mathcal{L}^{2}\right| \Delta_{i} .
$$

Notice that $\operatorname{supp}(\nu) \subset \operatorname{supp}(\mu) \subset F$. Moreover, we have $\nu\left(Q_{i}\right)=\left\langle\pi^{-1} \bar{\partial} f, g_{i}\right\rangle$, and since $\sum_{i \in I} g_{i}=1$ on $E$, we also have $\nu(F)=\sum_{i \in I} \nu\left(Q_{i}\right)=f^{\prime}(\infty) \geq$ $\gamma^{h}(E) / 2$ (which yields (f)).

We have $d \nu=b d \mu$, with $b=\left\langle\pi^{-1} \bar{\partial} f, g_{i}\right\rangle$ on $\Delta_{i}$. To estimate $\|b\|_{L^{\infty}(\mu)}$, notice that $\left\|V_{g_{i}} f\right\|_{\infty} \leq C$, and so

$$
\left|\left\langle\pi^{-1} \bar{\partial} f, g_{i}\right\rangle\right|=\left|\left(V_{g_{i}} f\right)^{\prime}(\infty)\right| \leq C \gamma^{h}\left(E \cap 2 Q_{i}\right)=C h\left(r_{i}\right)=C \mu\left(\Delta_{i}\right) .
$$

As a consequence, $\|b\|_{L^{\infty}(\mu)} \leq C$, and (e) is proved. 
It remains to check that (d) also holds. Using (7.4), the assumption $\gamma^{h}\left(E \cap 2 Q_{i}\right) \leq A \gamma_{+}^{h}\left(E \cap 2 Q_{i}\right)$, (b), and the hypothesis $A \gamma_{+}^{h}(E) \leq \gamma^{h}(E)$, we obtain the following inequalities:

$$
\begin{aligned}
\gamma^{h}(E) \leq 2\left|f^{\prime}(\infty)\right| & =\left|\sum_{i \in I}\left\langle\pi^{-1} \bar{\partial} f, g_{i}\right\rangle\right| \leq \sum_{i \in I}\left|\left\langle\pi^{-1} \bar{\partial} f, g_{i}\right\rangle\right| \\
& \leq C \sum_{i \in I} \gamma^{h}\left(E \cap 2 Q_{i}\right)=C \mu(F) \\
& \leq C A \sum_{i \in I} \gamma_{+}^{h}\left(E \cap 2 Q_{i}\right) \leq C A \gamma_{+}^{h}(E) \leq C \gamma^{h}(E),
\end{aligned}
$$

which gives (d) (with constants independent of $A$ ).

7.6. Construction of the exceptional set $H$ and proof of (i)-(j). Let $C_{h}$ be some big constant to be fixed below. Following [NTV2], given $x \in F$, $r>0$, we say that $B(x, r)$ is a non Ahlfors disk if $\mu(B(x, r))>C_{h} h(r)$. For a fixed $x \in F$, if there exists some $r>0$ such that $B(x, r)$ is a non Ahlfors disk, then we say that $x$ is a non Ahlfors point. For any $x \in F$, we denote

$$
\mathcal{R}(x)=\sup \{r>0: B(x, r) \text { is a non Ahlfors disk }\} .
$$

If $x \in F$ is an Ahlfors point, we set $\mathcal{R}(x)=0$. We say that $\mathcal{R}(x)$ is the Ahlfors radius of $x$.

Observe (d) implies that $\mu(F) \leq C_{a} \gamma^{h}(E) \leq C_{a} \gamma^{h}(F) \leq C_{14} h(\operatorname{diam}(F))$. Therefore,

$$
\mu(B(x, r)) \leq \mu(F) \leq C_{14} h(\operatorname{diam}(F)) \leq C_{15} h(r)
$$

for $r \geq \operatorname{diam}(F) / 100$. Thus $\mathcal{R}(x) \leq \operatorname{diam}(F) / 100$ for all $x \in F$, if we choose $C_{h} \geq C_{15}$.

We denote

$$
H_{0}=\bigcup_{x \in F, \mathcal{R}(x)>0} B(x, \mathcal{R}(x)) .
$$

By Vitali's $5 r$-Covering Theorem there is a disjoint subfamily $\left\{B\left(x_{k}, \mathcal{R}\left(x_{k}\right)\right)\right\}_{k}$ such that $H_{0} \subset \bigcup_{k} B\left(x_{k}, 5 \mathcal{R}\left(x_{k}\right)\right)$. We denote

$$
H=\bigcup_{k} B\left(x_{k}, 5 \mathcal{R}\left(x_{k}\right)\right) \text {. }
$$

Since $H_{0} \subset H$, all non Ahlfors disks are contained in $H$ and then,

$$
\operatorname{dist}(x, F \backslash H) \geq \mathcal{R}(x)
$$

for all $x \in F$.

Since $\mu\left(B\left(x_{k}, \mathcal{R}\left(x_{k}\right)\right)\right) \geq C_{h} h\left(\mathcal{R}\left(x_{k}\right)\right)$ for every $k$, we get

$$
\sum_{k} h\left(5 \mathcal{R}\left(x_{k}\right)\right) \leq C \sum_{k} h\left(\mathcal{R}\left(x_{k}\right)\right) \leq \frac{C}{C_{h}} \sum_{k} \mu\left(B\left(x_{k}, \mathcal{R}\left(x_{k}\right)\right)\right) \leq \frac{C}{C_{h}} \mu(F),
$$

with $\frac{C}{C_{h}}$ arbitrarily small (choosing $C_{h}$ big enough). 
7.7. Proof of (g) in First Main Lemma. In this subsection we will show that

$$
\int_{F \backslash H} \mathcal{C}_{*} \nu d \mu \leq C_{c} \mu(F) .
$$

We will work with the regularized operators $K_{\varepsilon}$ introduced in Section 2 . Remember that $\left|\mathcal{C}\left(\pi^{-1} \bar{\partial} f\right)(z)\right| \leq 1 \mathcal{L}^{2}$-a.e. $z \in \mathbb{C}$. Thus,

$$
\left\|K_{\varepsilon}\left(\pi^{-1} \bar{\partial} f\right)\right\|_{L^{\infty}}=\left\|\psi_{\varepsilon} * \mathcal{C}\left(\pi^{-1} \bar{\partial} f\right)\right\|_{L^{\infty}} \leq 1
$$

and so $K_{*}\left(\pi^{-1} \bar{\partial} f\right)(z) \leq 1$ for all $z \in \mathbb{C}, \varepsilon>0$.

To estimate $K_{*} \nu$, we will deal with the term $K_{*}\left(\nu-\pi^{-1} \bar{\partial} f\right)$. This will be the main point for the proof of (7.8).

We denote $\nu_{i}:=\nu \mid Q_{i}$.

Lemma 7.3. For every $z \in \mathbb{C} \backslash 10 Q_{i}$, we have

$$
K_{*}\left(\nu_{i}-\pi^{-1} g_{i} \bar{\partial} f\right)(z) \leq \frac{C \ell\left(Q_{i}\right) \mu\left(Q_{i}\right)}{\operatorname{dist}\left(z, 2 Q_{i}\right)^{2}}
$$

Proof. We set $\alpha_{i}=\nu_{i}-\pi^{-1} g_{i} \bar{\partial} f$. Notice that $\alpha_{i}$ is a distribution supported on $\left(E \cap 2 Q_{i}\right) \cup \Delta_{i}$. We have to show that

$$
\left|K_{\varepsilon} \alpha_{i}(z)\right| \leq \frac{C \ell\left(Q_{i}\right) \mu\left(Q_{i}\right)}{\operatorname{dist}\left(z, 2 Q_{i}\right)^{2}}
$$

for all $\varepsilon>0$.

Assume first $\varepsilon \leq \operatorname{dist}\left(z, 2 Q_{i}\right) / 2$. Since $\left|\mathcal{C} \alpha_{i}(w)\right| \leq C$ for all $w \notin \operatorname{supp}\left(\alpha_{i}\right)$ and $\left\langle\alpha_{i}, 1\right\rangle=0$, we have

$$
\left|\mathcal{C} \alpha_{i}(w)\right| \leq \frac{C \operatorname{diam}\left(\operatorname{supp}\left(\alpha_{i}\right)\right) \gamma^{h}\left(\operatorname{supp}\left(\alpha_{i}\right)\right)}{\operatorname{dist}\left(w, \operatorname{supp}\left(\alpha_{i}\right)\right)^{2}}
$$

by Lemma 5.5. On the other hand, by Lemma 5.3, we have

$$
\gamma^{h}\left(\operatorname{supp}\left(\alpha_{i}\right)\right) \leq \gamma^{h}\left(\left(E \cap 2 Q_{i}\right) \cup \Delta_{i}\right) \leq C\left(\gamma^{h}\left(\Delta_{i}\right)+\gamma^{h}\left(E \cap 2 Q_{i}\right)\right) .
$$

Therefore, by the definition of $\Delta_{i}$, we get

$$
\gamma^{h}\left(\Delta_{i} \cup\left(E \cap 2 Q_{i}\right)\right) \leq C \gamma^{h}\left(E \cap 2 Q_{i}\right)=C \mu\left(Q_{i}\right) .
$$

If $w \in B(z, \varepsilon)$, then $\operatorname{dist}\left(w, 2 Q_{i}\right) \approx \operatorname{dist}\left(z, 2 Q_{i}\right)$. By (7.11) and (7.12) we obtain

$$
\left|\mathcal{C} \alpha_{i}(w)\right| \leq \frac{C \ell\left(Q_{i}\right) \mu\left(Q_{i}\right)}{\operatorname{dist}\left(z, 2 Q_{i}\right)^{2}}
$$

Making the convolution with $\psi_{\varepsilon},(7.10)$ follows for $\varepsilon \leq \operatorname{dist}\left(z, 2 Q_{i}\right) / 2$.

Suppose now that $\varepsilon>\operatorname{dist}\left(z, 2 Q_{i}\right) / 2$. We denote $h_{i}=\psi_{\varepsilon} * \alpha_{i}$. Then we have

Therefore,

$$
K_{\varepsilon} \alpha_{i}=\psi_{\varepsilon} * \frac{1}{z} * \alpha_{i}=\mathcal{C}\left(h_{i} d \mathcal{L}^{2}\right)
$$

$$
\left|K_{\varepsilon} \alpha_{i}(z)\right| \leq \int \frac{\left|h_{i}(\xi)\right|}{|\xi-z|} d \mathcal{L}^{2}(\xi) \leq C\left\|h_{i}\right\|_{\infty}\left[\mathcal{L}^{2}\left(\operatorname{supp}\left(h_{i}\right)\right)\right]^{1 / 2} .
$$


We have to estimate $\left\|h_{i}\right\|_{\infty}$ and $\mathcal{L}^{2}\left(\operatorname{supp}\left(h_{i}\right)\right)$. Observe that, if we write $\ell_{i}=\ell\left(Q_{i}\right)$ and we denote the center of $Q_{i}$ by $z_{i}$, we get

$$
\operatorname{supp}\left(h_{i}\right) \subset \operatorname{supp}\left(\psi_{\varepsilon}\right)+\operatorname{supp}\left(\alpha_{i}\right) \subset B(0, \varepsilon)+B\left(z_{i}, 2 \ell_{i}\right)=B\left(z_{i}, \varepsilon+2 \ell_{i}\right) .
$$

Thus, $\mathcal{L}^{2}\left(\operatorname{supp}\left(h_{i}\right)\right) \leq C \varepsilon^{2}$, since $\ell_{i} \leq \varepsilon\left(\right.$ recall $\left.\left.z \notin 10 Q_{i}, \varepsilon>\operatorname{dist}\left(z, 2 Q_{i}\right) / 2\right)\right)$.

Let us deal with $\left\|h_{i}\right\|_{\infty}$ now. Let $\eta_{i}$ be a $\mathcal{C}^{\infty}$ function supported on $3 Q_{i}$ which is identically 1 on $2 Q_{i}$ and such that $\left\|\nabla \eta_{i}\right\|_{\infty} \leq C / \ell_{i}$. Since $\left\langle\alpha_{i}, 1\right\rangle=0$, we have

$$
\begin{array}{r}
h_{i}(w)=\left(\psi_{\varepsilon} * \alpha_{i}\right)(w)=\left\langle\alpha_{i}, \psi_{\varepsilon}(\cdot-w)\right\rangle=\left\langle\alpha_{i}, \psi_{\varepsilon}(\cdot-w)-\psi_{\varepsilon}\left(z_{i}-w\right)\right\rangle \\
=\frac{\ell_{i}}{\varepsilon^{3}}\left\langle\alpha_{i}, \frac{\varepsilon^{3}}{\ell_{i}}\left(\psi_{\varepsilon}(\cdot-w)-\psi_{\varepsilon}\left(z_{i}-w\right)\right) \eta_{i}\right\rangle=: \frac{\ell_{i}}{\varepsilon^{3}}\left\langle\alpha_{i}, \varphi_{w} \eta_{i}\right\rangle .
\end{array}
$$

We will show below that $C^{-1} \mathcal{C}\left(\varphi_{w} \eta_{i} \alpha_{i}\right) \in A^{h}\left(\operatorname{supp}\left(\alpha_{i}\right)\right)$ for some constant $C$. Assuming this fact for the moment, by (7.12) we deduce

$\left|\frac{\ell_{i}}{\varepsilon^{3}}\left\langle\alpha_{i}, \varphi_{w} \eta_{i}\right\rangle\right|=\left|\frac{\ell_{i}}{\varepsilon^{3}} \mathcal{C}\left(\varphi_{w} \eta_{i} \alpha_{i}\right)^{\prime}(\infty)\right| \leq \frac{C \ell_{i}}{\varepsilon^{3}} \gamma^{h}\left(\Delta_{i} \cup\left(E \cap 2 Q_{i}\right)\right) \leq \frac{C \ell_{i} \mu\left(Q_{i}\right)}{\varepsilon^{3}}$.

Therefore,

$$
\left\|h_{i}\right\|_{\infty} \leq \frac{C \ell_{i} \mu\left(Q_{i}\right)}{\varepsilon^{3}}
$$

By (7.13) and the estimates on $\left\|h_{i}\right\|_{\infty}$ and $\mathcal{L}^{2}\left(\operatorname{supp}\left(h_{i}\right)\right)$, we obtain

$$
\left|K_{\varepsilon} \alpha_{i}(z)\right| \leq \frac{C \ell\left(Q_{i}\right) \mu\left(Q_{i}\right)}{\varepsilon^{2}} \leq \frac{C \ell\left(Q_{i}\right) \mu\left(Q_{i}\right)}{\operatorname{dist}\left(z, 2 Q_{i}\right)^{2}} .
$$

It remains to prove that $C^{-1} \mathcal{C}\left(\varphi_{w} \eta_{i} \alpha_{i}\right) \in A^{h}\left(\operatorname{supp}\left(\alpha_{i}\right)\right)$. Observe that $\mathcal{C}\left(\varphi_{w} \eta_{i} \alpha_{i}\right)=V_{\left(\varphi_{w} \eta_{i}\right)}\left(\mathcal{C} \alpha_{i}\right)$ and remember that $\mathcal{C} \alpha_{i}$ is a bounded function. By Lemma 5.2, since $\operatorname{supp}\left(\varphi_{w} \eta_{i}\right) \subset 3 Q_{i}$, it is enough to show that

$$
\left\|\varphi_{w} \eta_{i}\right\|_{\infty} \leq C
$$

and

$$
\left\|\nabla\left(\varphi_{w} \eta_{i}\right)\right\|_{\infty} \leq \frac{C}{\ell_{i}}
$$

For $\xi \in 3 Q_{i}$, we have

$$
\left|\varphi_{w}(\xi)\right|=\frac{\varepsilon^{3}}{\ell_{i}}\left|\psi_{\varepsilon}(\xi-w)-\psi_{\varepsilon}\left(z_{i}-w\right)\right| \leq \varepsilon^{3}\left\|\nabla \psi_{\varepsilon}\right\|_{\infty} \leq C,
$$

which yields (7.14). Finally, (7.15) follows easily too:

$$
\left\|\nabla\left(\varphi_{w} \eta_{i}\right)\right\|_{\infty} \leq\left\|\nabla \varphi_{w}\right\|_{\infty}+\left\|\varphi_{w}\right\|_{\infty, 3 Q_{i}}\left\|\nabla \eta_{i}\right\|_{\infty} \leq \frac{C}{\ell_{i}} .
$$

We are done. 
Now we are ready to prove (7.8). We write

$$
\begin{aligned}
\int_{F \backslash H} K_{*} \nu d \mu & \leq \int_{F \backslash H} K_{*}\left(\pi^{-1} \bar{\partial} f\right) d \mu+\int_{F \backslash H} K_{*}\left(\nu-\pi^{-1} \bar{\partial} f\right) d \mu \\
& \leq C \mu(F \backslash H)+\sum_{i \in I} \int_{F \backslash H} K_{*}\left(\nu_{i}-\pi^{-1} g_{i} \bar{\partial} f\right) d \mu .
\end{aligned}
$$

To estimate the last sum of integrals we argue as in [To5]. Using Lemma 7.3 and recalling that $\left\|K_{*}\left(\nu_{i}-\pi^{-1} g_{i} \bar{\partial} f\right)\right\|_{L^{\infty}(\mu)} \leq C$, we get

$$
\int_{F \backslash H} K_{*}\left(\nu_{i}-\pi^{-1} g_{i} \bar{\partial} f\right) d \mu \leq C \mu\left(4 Q_{i}\right)
$$

(see the last part of Subsection 7.3 in [To5] for the details). Thus, by the finite overlap of the squares $4 Q_{i}, i \in I$, and (7.16), we get

$$
\int_{F \backslash H} K_{*} \nu d \mu \leq C \mu(F \backslash H)+C \sum_{i \in I} \mu\left(4 Q_{i}\right) \leq C \mu(F) .
$$

We also have

$$
\left|K_{*} \nu(z)-\mathcal{C}_{*} \nu(z)\right| \leq C M \nu(z) .
$$

By (e), if $z \in F \backslash H$, we have $M \nu(z) \leq C M \mu(z) \leq C M^{h} \mu(z) \leq C$. Thus (7.17) and (7.18) imply

$$
\int_{F \backslash H} \mathcal{C}_{*} \nu(z) d \mu(z) \leq C \mu(F) .
$$

7.8. Proof of (h) in First Main Lemma. For a given function $\varphi \in \mathcal{C}^{\infty}$ supported on some ball $B_{r}$ of radius $r$, we have to show that

$$
\left|\int \varphi d \nu\right| \leq C h(r) r\|\nabla \varphi\| .
$$

First, assume that there exists some square $Q_{i_{0}}, i_{0} \in I$, with side length $\ell\left(Q_{i_{0}}\right) \geq r$ such that $Q_{i_{0}} \cap B_{r} \neq \varnothing$. Then we have $B_{r} \subset 5 Q_{i_{0}}$. Thus,

$$
\#\left\{i: Q_{i} \cap \operatorname{supp}(\varphi) \neq \varnothing\right\} \leq C .
$$

Remember that for each square $Q_{i}, \mu\left(Q_{i}\right)=C \gamma^{h}\left(E \cap 2 Q_{i}\right) \leq C h\left(\ell\left(Q_{i}\right)\right)$. Then, since $\nu=b d \mu$, with $b$ bounded, we get

$$
\left|\int_{Q_{i}} \varphi d \nu\right| \leq C \mu\left(Q_{i} \cap B_{r}\right)\|\varphi\|_{\infty} \leq C \mu\left(Q_{i} \cap B_{r}\right) r\|\nabla \varphi\|_{\infty} .
$$

Remember that $\mu\left|Q_{i}=h\left(r_{i}\right) \mathcal{L}^{2}\right| \Delta_{i} / \mathcal{L}^{2}\left(\Delta_{i}\right)$, where $\Delta_{i}$ is a closed disk of radius $r_{i}$. Using $\mu\left(Q_{i} \cap B_{r}\right) \leq C \min \left(r^{2}, r_{i}^{2}\right) h\left(r_{i}\right) / r_{i}^{2}$, it follows easily that

$$
\left|\int_{Q_{i}} \varphi d \nu\right| \leq C h(r) r\|\nabla \varphi\|_{\infty} .
$$

From (7.20), we deduce that (7.19) holds in this case. 
Suppose now that all the squares $Q_{i}, i \in I$, that intersect $B_{r}$ satisfy $\ell\left(Q_{i}\right)<r$. We will prove below that for any collection of points $\left\{z_{i}\right\}_{i \in I}$ with $z_{i} \in Q_{i}$

$$
\left|\sum_{i \in I} \varphi\left(z_{i}\right) \nu\left(Q_{i}\right)+\frac{1}{\pi} \int f \bar{\partial} \varphi d \mathcal{L}^{2}\right| \leq C h(r) r\|\nabla \varphi\|_{\infty} .
$$

Notice that this estimate implies that

$$
\left|\sum_{i \in I} \varphi\left(z_{i}\right) \nu\left(Q_{i}\right)\right| \leq C h(r) r\|\nabla \varphi\|_{\infty}
$$

because $f \in A^{h}(E)$. Since $\nu_{\mid Q_{i}}$ is a constant multiple of a positive measure, by continuity there exists some point $z_{i} \in Q_{i}$ such that $\varphi\left(z_{i}\right) \nu\left(Q_{i}\right)=$ $\int_{Q_{i}} \varphi d \nu$. From this fact and the preceding inequality, (7.19) follows.

To prove (7.21), we set

$$
\left|\sum_{i \in I} \varphi\left(z_{i}\right) \nu\left(Q_{i}\right)+\frac{1}{\pi} \int f \bar{\partial} \varphi d \mathcal{L}^{2}\right| \leq \sum_{i \in I}\left|\varphi\left(z_{i}\right) \nu\left(Q_{i}\right)+\frac{1}{\pi} \int V_{g_{i}} f \bar{\partial} \varphi d \mathcal{L}^{2}\right| .
$$

For each $i$ we have

$$
\begin{aligned}
\left|\varphi\left(z_{i}\right) \nu\left(Q_{i}\right)+\int V_{g_{i}} f \bar{\partial} \varphi d \mathcal{L}^{2}\right| & =\frac{1}{\pi}\left|-\varphi\left(z_{i}\right) \int f \bar{\partial} g_{i} d \mathcal{L}^{2}+\int f \bar{\partial}\left(\varphi g_{i}\right) d \mathcal{L}^{2}\right| \\
& =\frac{1}{\pi}\left|\int f \bar{\partial}\left(\left[\varphi-\varphi\left(z_{i}\right)\right] g_{i}\right) d \mathcal{L}^{2}\right|
\end{aligned}
$$

We denote $\psi_{i}:=\left[\varphi-\varphi\left(z_{i}\right)\right] g_{i}$ and $\ell_{i}=\ell\left(Q_{i}\right)$. Because $f \in A^{h}(E)$, the left side of (7.23) is bounded above by

$$
\begin{aligned}
C h\left(\ell_{i}\right) \ell_{i}\left\|\nabla \psi_{i}\right\|_{\infty} & \leq C h\left(\ell_{i}\right) \ell_{i}\left(\left\|\nabla g_{i}\right\|_{\infty}\left\|\varphi-\varphi\left(z_{i}\right)\right\|_{\infty, 2 Q_{i}}+\left\|g_{i}\right\|_{\infty}\|\nabla \varphi\|_{\infty}\right) \\
& \leq C h\left(\ell_{i}\right) \ell_{i}\|\nabla \varphi\|_{\infty} .
\end{aligned}
$$

Taking into account that $h(t) / t$ is non decreasing in $t$ we obtain

$$
\begin{aligned}
\left|\sum_{i \in I} \varphi\left(z_{i}\right) \nu\left(Q_{i}\right)+\frac{1}{\pi} \int f \bar{\partial} \varphi d \mathcal{L}^{2}\right| & \leq C \sum_{i: Q_{i} \cap B_{r} \neq \varnothing} h\left(\ell_{i}\right) \ell_{i}\|\nabla \varphi\|_{\infty} \\
& \leq C \sum_{i: Q_{i} \cap B_{r} \neq \varnothing} \frac{h(r)}{r} \ell_{i}^{2}\|\nabla \varphi\|_{\infty} \\
& \leq C \frac{h(r)}{r} \mathcal{L}^{2}\left(B_{3 r}\right)\|\nabla \varphi\|_{\infty} \\
& =C h(r) r\|\nabla \varphi\|_{\infty},
\end{aligned}
$$

and we are done. 
7.9. The dyadic exceptional set $H_{\mathcal{D}}$. Remember that in (7.5) we defined $H=\bigcup_{k} B\left(x_{k}, 5 \mathcal{R}\left(x_{k}\right)\right)$, where $\left\{B\left(x_{k}, \mathcal{R}\left(x_{k}\right)\right)\right\}_{k}$ is some precise family of non Ahlfors disks. For technical reasons, it is convenient to introduce a dyadic version of $H$ made up of dyadic squares. Let $\mathcal{D}$ be a fixed dyadic lattice and consider the family $\mathcal{D}_{H} \subset \mathcal{D}$ of dyadic squares such that $R \in \mathcal{D}_{H}$ if there exists some ball $B\left(x_{k}, 5 \mathcal{R}\left(x_{k}\right)\right)$ satisfying

$$
B\left(x_{k}, 5 \mathcal{R}\left(x_{k}\right)\right) \cap R \neq \varnothing
$$

and

$$
10 \mathcal{R}\left(x_{k}\right)<\ell(R) \leq 20 \mathcal{R}\left(x_{k}\right)
$$

Notice that

$$
\bigcup_{k} B\left(x_{k}, 5 \mathcal{R}\left(x_{k}\right)\right) \subset \bigcup_{R \in \mathcal{D}_{H}} R .
$$

We take a subfamily of disjoint maximal squares $\left\{R_{j}\right\}_{j \in I_{H}}$ from $\mathcal{D}_{H}$ such that

$$
\bigcup_{R \in \mathcal{D}_{H}} R=\bigcup_{j \in I_{H}} R_{j}
$$

and we define the dyadic exceptional set $H_{\mathcal{D}}$ as

$$
H_{\mathcal{D}}=\bigcup_{j \in I_{H}} R_{k}
$$

Observe that (7.26) implies $H \subset H_{\mathcal{D}}$ and, since for each ball $B\left(x_{k}, 5 \mathcal{R}\left(x_{k}\right)\right)$ there are at most four squares $R \in \mathcal{D}_{H}$ satisfying (7.24) and (7.25), by (7.7), we obtain

$\sum_{j \in I_{H}} h\left(\ell\left(R_{j}\right)\right) \leq 4 \sum_{k} h\left(20 \mathcal{R}\left(x_{k}\right)\right) \leq C_{15} \sum_{k} h\left(\mathcal{R}\left(x_{k}\right)\right) \leq \frac{C_{15}}{C_{h}} \mu(F) \leq \varepsilon \mu(F)$, assuming $C_{h} \geq C_{15} \varepsilon^{-1}$.

7.10. The accretivity condition and the exceptional set $T_{\mathcal{D}}$. We say that a square $R \subset \mathbb{C}$ is accretive if

$$
\mu(R) \leq C_{d}|\nu(R)|
$$

where $C_{d}$ is some big constant to be chosen later. We denote by $\mathcal{D}_{T}$ the collection of non accretive squares from $\mathcal{D}$. Let $\left\{R_{k}\right\}_{k \in I_{T}} \subset \mathcal{D}_{T}$ be the subfamily of disjoint maximal dyadic squares from $\mathcal{D}_{T}$. The exceptional set $T_{\mathcal{D}}$ is

$$
T_{\mathcal{D}}=\bigcup_{k \in I_{T}} R_{k}
$$

From the properties (d) and (f), one can easily infer that $\left|\nu\left(T_{\mathcal{D}}\right)\right| \leq \frac{1}{2}|\nu(F)|$ if the constant $C_{d}$ in (7.28) is chosen big enough. However, for technical reasons (as in [To5]), we need to estimate the size of both $H_{\mathcal{D}}$ and $T_{\mathcal{D}}$ simultaneously. Roughly speaking, $H_{\mathcal{D}}$ is a small set because the measure $\nu$ 
is "not too concentrated", that is, because of (h) in First Main Lemma. If we knew that

$$
|\nu(Q)| \leq C h(\ell(Q)) \quad \text { for every square } Q \subset \mathbb{C},
$$

then we would try to argue as in Section 8 of [To5] in order to show that $\left|\nu\left(H_{\mathcal{D}}\right)\right|$ and $\mu\left(H_{\mathcal{D}}\right)$ are small. However, instead of $(7.29)$, we only know (h), which is a weaker condition. In Section 7.12, instead of estimating $\left|\nu\left(H_{\mathcal{D}} \cup T_{\mathcal{D}}\right)\right|$ and $\mu\left(H_{\mathcal{D}} \cup T_{\mathcal{D}}\right)$ for a particular dyadic lattice $\mathcal{D}$, we will prove that the average values of $\left|\nu\left(H_{\mathcal{D}} \cup T_{\mathcal{D}}\right)\right|$ and $\mu\left(H_{\mathcal{D}} \cup T_{\mathcal{D}}\right)$ are small when we take different dyadic lattices $\mathcal{D}$.

7.11. Random dyadic lattices. We are going to introduce random dyadic lattices. We follow the construction of [NTV2].

Suppose that $F \subset B\left(0,2^{N-3}\right)$, where $N$ is a big enough integer. Consider the random square $Q^{0}(w)=w+\left[-2^{N}, 2^{N}\right)^{2}$, with $w \in\left[-2^{N-1}, 2^{N-1}\right)^{2}=$ : $\Omega$. We take $Q^{0}(w)$ as the starting square of the dyadic lattice $\mathcal{D}(w)$. Observe that $F \subset Q^{0}(w)$ for all $w \in \Omega$. Only the dyadic squares which are contained in $Q^{0}(w)$ will play some role in the arguments below. For the moment, we don't worry about the other squares.

We take a uniform probability on $\Omega$. So we let the probability measure $P$ be the normalized Lebesgue measure on the square $\Omega$.

A square $Q \in \mathcal{D} \equiv \mathcal{D}(w)$ contained in $Q^{0}(w)$ is called terminal if $Q \subset$ $H_{\mathcal{D}} \cup T_{\mathcal{D}}$. Otherwise, it is called transit. The set of terminal squares is denoted by $\mathcal{D}^{\text {term }}$, and the set of transit squares by $\mathcal{D}^{t r}$. It is easy to check that $Q^{0}$ is always transit.

7.12. The size of $H_{\mathcal{D}} \cup T_{\mathcal{D}}$. The sets $H_{\mathcal{D}}$ and $T_{\mathcal{D}}$ are made up of dyadic squares. For each dyadic lattice $\mathcal{D}(w), w \in \Omega$, we obtain the corresponding sets $H_{\mathcal{D}}(w)$ and $T_{\mathcal{D}}(w)$. Notice that for all $w \in \Omega$, we have $H \subset H_{\mathcal{D}}(w)$.

Lemma 7.4. If $C_{h}$ and $C_{d}$ have been chosen big enough, then

$$
\int_{w \in \Omega}\left|\nu\left(H_{\mathcal{D}}(w) \cup T_{\mathcal{D}}(w)\right)\right| d P(w) \leq \frac{1}{2}|\nu(F)|
$$

and

$$
\int_{w \in \Omega} \mu\left(H_{\mathcal{D}}(w) \cup T_{\mathcal{D}}(w)\right) \mid d P(w) \leq \delta_{0} \mu(F),
$$

where $\delta_{0}<1$ is an absolute constant.

Proof. First we will prove (7.30). Let $\left\{R_{k}(w)\right\}_{k \in I_{H T}(w)}$ be the subfamily of different maximal (and thus disjoint) squares from

$$
\left\{R_{k}(w)\right\}_{k \in I_{H}(w)} \cup\left\{R_{k}(w)\right\}_{k \in I_{T}(w)},
$$

so that

$$
H_{\mathcal{D}}(w) \cup T_{\mathcal{D}}(w)=\bigcup_{k \in I_{H T}(w)} R_{k}(w)
$$


For each $w \in \Omega$, we have

$$
\begin{aligned}
\left|\nu\left(H_{\mathcal{D}}(w) \cup T_{\mathcal{D}}(w)\right)\right| & \leq \sum_{k \in I_{H T}(w)}\left|\nu\left(R_{k}(w)\right)\right| \\
& \leq \sum_{k \in I_{H}(w)}\left|\nu\left(R_{k}(w)\right)\right|+\sum_{k \in I_{T}(w)}\left|\nu\left(R_{k}(w)\right)\right| .
\end{aligned}
$$

The last sum above is easy to estimate using (7.28):

$$
\begin{aligned}
\sum_{k \in I_{T}(w)}\left|\nu\left(R_{k}(w)\right)\right| & \leq C_{d}^{-1} \sum_{k \in I_{T}(w)} \mu\left(R_{k}(w)\right) \\
& \leq C_{d}^{-1} \mu(F) \leq 2 C_{a} C_{d}^{-1}|\nu(F)| \leq \frac{1}{4}|\nu(F)|,
\end{aligned}
$$

for $C_{d}$ big enough.

To estimate $\left|\nu\left(H_{\mathcal{D}}(w)\right)\right|$, we consider for each square $R_{k}(w), k \in I_{H}(w)$, a $\mathcal{C}^{\infty}$ function $\varphi_{k}^{w}$ supported on $(1+\eta) R_{k}(w)$ (with $0<\eta \leq 1 / 10$ to be chosen later), such that $0 \leq \varphi_{k}^{w} \leq 1, \varphi_{k}^{w}$ identically 1 on $R_{k}(w)$, satisfying $\left\|\nabla \varphi_{k}^{w}\right\|_{\infty} \leq C \eta^{-1} \ell\left(R_{k}(w)\right)^{-1}$. Using the fact that $d \nu=b d \mu$, with $b$ bounded, and (h), we get

$$
\begin{aligned}
\left|\nu\left(R_{k}(w)\right)\right| & \leq\left|\int \varphi_{k}^{w} d \nu\right|+C \mu\left((1+\eta) R_{k}(w) \backslash R_{k}(w)\right) \\
& \leq \frac{C}{\eta} h\left(\ell\left(R_{k}(w)\right)\right)+C \mu\left((1+\eta) R_{k}(w) \backslash R_{k}(w)\right) .
\end{aligned}
$$

Therefore, by (7.27) we obtain

$$
\sum_{k \in I_{H}(w)}\left|\nu\left(R_{k}(w)\right)\right| \leq \frac{C}{C_{h} \eta} \mu(F)+C \sum_{k \in I_{H}(w)} \mu\left((1+\eta) R_{k}(w) \backslash R_{k}(w)\right) .
$$

We will show in Lemma 7.5 below that

$$
\int_{w \in \Omega} \sum_{k \in I_{H}(w)} \mu\left((1+\eta) R_{k}(w) \backslash R_{k}(w)\right) \leq C_{16} \eta \mu(F),
$$

where $C_{16}$ is an absolute constant. As a consequence,

$$
\int_{w \in \Omega} \sum_{k \in I_{H}(w)}\left|\nu\left(R_{k}(w)\right)\right| d P(w) \leq C_{17}\left(\frac{1}{\eta C_{h}}+\eta\right)|\nu(F)| .
$$

Therefore, if $\eta \leq 1 / 8 C_{17}$ and $C_{h}$ is such that $C_{17} /\left(\eta C_{h}\right) \leq 1 / 8$, then

$$
\int_{w \in \Omega} \sum_{k \in I_{H}(w)}\left|\nu\left(R_{k}(w)\right)\right| d P(w) \leq|\nu(F)| / 4 .
$$

Inequality (7.30) follows from this estimated and (7.33). 
Let us see now how (7.31) is derived from (7.30). We have

$$
\begin{aligned}
\int_{w \in \Omega} \mid \nu(F \backslash & \left.\left(H_{\mathcal{D}}(w) \cup T_{\mathcal{D}}(w)\right)\right) \mid d P(w) \\
& \geq \int_{w \in \Omega}\left(|\nu(F)|-\left|\nu\left(H_{\mathcal{D}}(w) \cup T_{\mathcal{D}}(w)\right)\right|\right) d P(w) \geq \frac{1}{2}|\nu(F)| .
\end{aligned}
$$

Thus,

$$
\begin{aligned}
\mu(F) & \leq C \gamma^{h}(E) \leq C|\nu(F)| \leq C \int_{w \in \Omega}\left|\nu\left(F \backslash\left(H_{\mathcal{D}}(w) \cup T_{\mathcal{D}}(w)\right)\right)\right| d P(w) \\
& \leq C_{18} \int_{w \in \Omega} \mu\left(F \backslash\left(H_{\mathcal{D}}(w) \cup T_{\mathcal{D}}(w)\right)\right) d P(w) .
\end{aligned}
$$

Therefore,

$$
\int_{w \in \Omega} \mu\left(H_{\mathcal{D}}(w) \cup T_{\mathcal{D}}(w)\right) d P(w) \leq\left(1-\frac{1}{C_{18}}\right) \mu(F)=: \delta_{0} \mu(F) .
$$

It remains to prove the following result.

Lemma 7.5. Let $\eta$ be some constant with $0<\eta \leq 1 / 10$. Then,

$$
\int_{w \in \Omega} \sum_{k \in I_{H}(w)} \mu\left((1+\eta) R_{k}(w) \backslash R_{k}(w)\right) \leq C_{16} \eta \mu(F) .
$$

Proof. Let $B\left(x_{0}, r_{0}\right)$ be one of the balls $B\left(x_{j}, r_{j}\right)$ that form the exceptional set $H$, as explained in $(\mathrm{j})$ from First Main Lemma. Denote by $R_{1}(w), \ldots, R_{4}(w)$ the squares from $\mathcal{D}(w)$ such that $B\left(x_{0}, r_{0}\right) \subset \bigcup_{k=1}^{4} R_{k}(w)$, with $2 r_{0}<\ell\left(R_{k}(w)\right) \leq 4 r_{0}$ (remember (7.24) and (7.25)). Notice that $\bigcup_{k=1}^{4}(1+\eta) R_{k}(w) \subset B\left(x_{0}, 10 r_{0}\right)$, and since $B\left(x_{0}, \lambda r_{0}\right)$ is an Ahlfors disk for $\lambda>1$, unlike $B\left(x_{0}, r_{0} / 5\right)$, we have

$$
\mu\left(B\left(x_{0}, \lambda r_{0}\right)\right) \leq 100 \lambda^{2} h\left(r_{0} / 5\right) \leq 100 \lambda^{2} \mu\left(B\left(x_{0}, r_{0} / 5\right)\right) .
$$

We will show that

$$
\int_{w \in \Omega} \sum_{k=1}^{4} \mu\left((1+\eta) R_{k}(w) \backslash R_{k}(w)\right) d P(w) \leq C \eta \mu\left(B\left(x_{0}, r_{0} / 5\right)\right) .
$$

The lemma follows from this estimate and the fact that the balls $B\left(x_{j}, r_{j} / 5\right)$ are disjoint.

Let us prove (7.36) now. Let $S_{0}$ be a square centered at $x_{0}$ of side length $2^{m}$, with $m \in \mathbb{Z}$ such that

$$
B\left(x_{0}, 10 r_{0}\right) \subset S_{0} \subset B\left(x_{0}, 30 r_{0}\right) .
$$

Let $L_{V, t}$ be the vertical line $\{(x, y) \in \mathbb{C}: x=t\}$. Let $(a, b) \in \mathbb{C}$ be the lower left corner of $S_{0}$. By Fubini, it easily follows that for all $\varepsilon$ with $0<\varepsilon \leq 2^{m}$,

$$
\frac{1}{2^{m}} \int_{t \in\left(a, a+2^{m}\right]} \mu\left(U_{\varepsilon}\left(L_{V, t}\right) \cap S_{0}\right) d t \leq \frac{C \varepsilon}{2^{m}} \mu\left(2 S_{0}\right),
$$


where $U_{\varepsilon}\left(L_{V, t}\right)$ stands for the $\varepsilon$-neighborhood of $L_{V, t}$. The analogous estimate holds for horizontal lines $L_{H, t}$. Setting $\varepsilon=\eta \ell\left(R_{k}(w)\right)$, which depends neither on $k$ nor on $w$, we obtain

$$
\begin{aligned}
& \int_{w \in \Omega} \sum_{k=1}^{4} \mu\left((1+\eta) R_{k}(w) \backslash R_{k}(w)\right) d P(w) \\
& \leq \frac{C}{2^{m}} \int_{t \in\left(a, a+2^{m}\right]} \mu\left(U_{\varepsilon}\left(L_{V, t}\right) \cap S_{0}\right) d t+\frac{C}{2^{m}} \int_{t \in\left(b, b+2^{m}\right]} \mu\left(U_{\varepsilon}\left(L_{H, t}\right) \cap S_{0}\right) d t \\
& \leq \frac{C \eta \ell\left(R_{k}(w)\right)}{2^{m}} \mu\left(2 S_{0}\right) \leq C \eta \mu\left(B\left(x_{0}, r_{0} / 5\right)\right),
\end{aligned}
$$

by $(7.35)$.

7.13. The Second Main Lemma and the $T b$ Theorem of Nazarov, Treil and Volberg. The next step consists of proving the following result. We use the same notation of First Main Lemma.

Lemma 7.6 (Second Main Lemma). Assume $\gamma_{+}^{h}(E) \leq C_{13} h(\operatorname{diam}(E))$, $\gamma^{h}(E) \geq A \gamma_{+}^{h}(E)$, and $\gamma^{h}\left(E \cap 2 Q_{i}\right) \leq A \gamma_{+}^{h}\left(E \cap 2 Q_{i}\right)$ for all $i \in I$. Then there exists some subset $G \subset F$, with $\mu(F) \leq C_{19} \mu(G)$, such that $\mu(G \cap B(x, r)) \leq$ $C_{h} h(r)$ for all $x \in G, r>0$, and the Cauchy transform is bounded on $L^{2}(\mu \mid G)$ with $\|\mathcal{C}\|_{L^{2}(\mu \mid G), L^{2}(\mu \mid G)} \leq C_{20}$. The constants $C_{h}, C_{13}, C_{19}, C_{20}$ are absolute constants, and do not depend on $A$.

To prove this lemma we only have to apply a suitable version of a $T b$ type theorem of Nazarov, Treil and Volberg [NTV2] to the measure $\mu$ and the function $b$ constructed in First Main Lemma. The precise version of the $T b$ theorem of Nazarov, Treil and Volberg that we need uses the random dyadic lattices $\mathcal{D}(w), w \in \Omega$, introduced in Section 7.11 and reads as follows.

Theorem 7.7 ([NTV2]). Let $\mu$ be a measure supported on $F \subset \mathbb{C}$. Suppose that there exist a complex measure $\nu$ and, for each $w \in \Omega$, two exceptional sets $H_{\mathcal{D}}(w)$ and $T_{\mathcal{D}}(w)$ made up of dyadic squares from $\mathcal{D}(w)$ such that

(a) Every ball $B_{r}$ of radius $r$ such that $\mu\left(B_{r}\right)>C_{h} r$ is contained in $H_{\mathcal{D}}(w)$, for all $w \in \Omega$.

(b) $d \nu=b d \mu$, with $\|b\|_{L^{\infty}(\mu)} \leq C$.

(c) $\int_{\mathbb{C} \backslash H_{\mathcal{D}}(w)} \mathcal{C}_{*} \nu d \mu \leq C \mu(F)$, for all $w \in \Omega$.

(d) If $Q \in \mathcal{D}(w)$ is such that $Q \not \subset T_{\mathcal{D}(w)}$, then $\mu(Q) \leq C_{d}|\nu(Q)|$ (i.e. $Q$ is an accretive square).

(e) $\int_{w \in \Omega} \mu\left(H_{\mathcal{D}}(w) \cup T_{\mathcal{D}}(w)\right) d P(w) \leq \delta_{0} \mu(F)$, for some $\delta_{0}<1$.

Then there exists a subset $G \subset F \backslash \bigcap_{w \in \Omega}\left(H_{\mathcal{D}}(w) \cup T_{\mathcal{D}}(w)\right)$ such that the Cauchy transform is bounded on $L^{2}(\mu \mid G)$, with the $L^{2}$ norm bounded above by some constant depending on the constants above.

This theorem is not stated in [NTV2]. However, it can be deduced from the arguments in [NTV2]. Theorem 7.7, changing the statement (e) by

(e') $\mu\left(H_{\mathcal{D}}(w) \cup T_{\mathcal{D}}(w)\right) \leq \delta_{0} \mu(F)$, for all $w \in \Omega$ and some $\delta_{0}<1$, 
was used in [To5]. In this article, the reader will find the proof of the necessary modifications in order to derive Theorem 7.7 (changing (e) by $\left.\left(e^{\prime}\right)\right)$ from the results of [NTV2].

The only change needed in the arguments of [To5] in order to prove Theorem 7.7 with the statement (e) instead of (e') is a minor modification in the probabilistic argument explained in [To5, Section 11.5] (which, in its turn, describes the arguments of [NTV2, Section XXIII]). The reader can easily verify that the estimates of [To5, Section 11.5] (or [NTV2, Section XXIII]) hold if one assumes (e) instead of (e'). We will not go into more details.

Proof of Second Main Lemma. This is a direct consequence of First Main Lemma and Theorem 7.7.

Notice that the set $G$ obtained in Theorem 7.7 fulfills

$$
G \subset \mathbb{C} \backslash \bigcap_{w \in \Omega} H_{\mathcal{D}} \subset \mathbb{C} \backslash H
$$

and so $\mu(B(x, r)) \leq C_{h} h(r)$ for all $x \in G$.

7.14. The induction argument for the proof of Theorem 7.1. The arguments in this subsection are quite similar to the ones of [To5, Section 12]. The reader can check that the main differences with respect to [To5, Section 12] appear after Lemmas 7.8 and 7.9, and are due to the fact that now we are assuming that $E$ is an arbitrary compact set, and not a finite union of segments, say, as in [To5].

Lemma 7.8. There exists some absolute constant $B$ such that if $A \geq 1$ is any fixed constant and

(a) $\gamma_{+}^{h}(E) \leq C_{13} h(\operatorname{diam}(E))$

(b) $\gamma^{h}(E \cap Q) \leq A \gamma_{+}^{h}(E \cap Q)$ for all squares $Q$ with $\operatorname{diam}(Q) \leq \operatorname{diam}(E) / 5$,

(c) $\gamma^{h}(E) \geq A \gamma_{+}^{h}(E)$,

then $\gamma^{h}(E) \leq B \gamma_{+}^{h}(E)$.

Proof. By First Main Lemma 7.2 and Second Main Lemma 7.6, there are sets $F, G$ and a measure $\mu$ supported on $F$ such that

(i) $E \subset F$ and $\gamma_{+}^{h}(E) \approx \gamma_{+}^{h}(F)$,

(ii) $\mu(F) \approx \gamma^{h}(E)$,

(iii) $G \subset F$ and $\mu(G) \geq C_{19}^{-1} \mu(F)$,

(iv) $\mu(G \cap B(x, r)) \leq C_{h} h(r)$ for all $x \in G, r>0$, and $\|\mathcal{C}\|_{L^{2}(\mu \mid G), L^{2}(\mu \mid G)} \leq$ $C_{20}$.

From (iv) and (iii), we get

$$
\gamma_{+}^{h}(F) \geq C^{-1} \mu(G) \geq C^{-1} \mu(F) .
$$

Then, by (ii), the preceding inequality, and (i),

$$
\gamma^{h}(E) \leq C \mu(F) \leq C \gamma_{+}^{h}(F) \leq B \gamma_{+}^{h}(E) .
$$


As a corollary we deduce:

Lemma 7.9. There exists some absolute constant $A_{0}$ such that if $\gamma^{h}(E \cap$ $Q) \leq A_{0} \gamma_{+}^{h}(E \cap Q)$ for all squares $Q$ with $\operatorname{diam}(Q) \leq \operatorname{diam}(E) / 5$, then $\gamma^{h}(E) \leq A_{0} \gamma_{+}^{h}(E)$.

Proof. If $\gamma_{+}^{h}(E)>C_{13} h(\operatorname{diam}(E))$, then we get $\gamma_{+}^{h}(E)>C_{21} \gamma^{h}(E)$, and we are done provided that $A_{0} \geq C_{21}^{-1}$. We set $A_{0}=\max \left(1, C_{21}^{-1}, B\right)$. If $\gamma_{+}^{h}(E) \leq C_{13} h(\operatorname{diam}(E))$, then we also have $\gamma^{h}(E) \leq A_{0} \gamma_{+}^{h}(E)$. Otherwise, we apply Lemma 7.8 and we deduce $\gamma^{h}(E) \leq B \gamma_{+}^{h}(E) \leq A_{0} \gamma_{+}^{h}(E)$, which is a contradiction.

Notice that any constant $A_{0} \geq \max \left(1, C_{21}^{-1}, B\right)$ works in the argument above. So Lemma 7.9 holds for any constant $A_{0}$ sufficiently big.

Proof of Theorem 7.1. By the approximation property (e) of Lemma 6.1, if $d>0$ is small enough, then $\gamma_{+}^{h}\left(\overline{U_{d}(E)}\right) \leq \gamma_{+}^{h}(E)+\varepsilon$, where $\varepsilon>0$ is some arbitrarily small constant. Consider the compact set $E_{0}$ made up of all the closed dyadic squares (from the usual standard dyadic lattice, say) of side length $d / 2$ that intersect $E$. We will show below that $\gamma^{h}\left(E_{0}\right) \leq C \gamma_{+}^{h}\left(E_{0}\right)$. From this inequality, since $E \subset E_{0} \subset \overline{U_{d}(E)}$, we get

$$
\gamma^{h}(E) \leq \gamma^{h}\left(E_{0}\right) \leq C \gamma_{+}^{h}\left(E_{0}\right) \leq C\left(\gamma_{+}^{h}(E)+\varepsilon\right),
$$

and then we are done.

To prove that $\gamma^{h}\left(E_{0}\right) \leq C \gamma_{+}^{h}\left(E_{0}\right)$, we are going to show by induction on $n$ that if $R$ is a closed rectangle with sides parallel to the axes and diameter $\leq 4^{n-1} d, n \geq 0$, then

$$
\gamma^{h}\left(R \cap E_{0}\right) \leq A_{0} \gamma_{+}^{h}\left(R \cap E_{0}\right) .
$$

Notice that if $\operatorname{diam}(R) \leq d / 4$, then $R$ can intersect at most four of the dyadic squares of side length $d$ that form $E_{0}$. In any case it is easy to check that $R \cap E_{0}$ is either a rectangle or a polygon which can be decomposed as the union of two closed rectangles $R_{1}, R_{2}$ (with $R_{1} \cap R_{2} \neq \varnothing$ ). In the first case, (7.37) follows from Lemma 5.1 (assuming $A_{0}$ sufficiently big); and in the second one, by Lemmas 5.3 and 5.1, we have

$\gamma^{h}\left(R_{1} \cup R_{2}\right) \leq C\left(\gamma^{h}\left(R_{1}\right)+\gamma^{h}\left(R_{2}\right)\right) \leq C\left(\gamma_{+}^{h}\left(R_{1}\right)+\gamma_{+}^{h}\left(R_{2}\right)\right) \leq 2 C \gamma_{+}^{h}\left(R_{1} \cup R_{2}\right)$.

Let us see now that if (7.37) holds for all rectangles $R$ with diameter $\leq 4^{n} d$, then it also holds for a rectangle $\widetilde{R}$ with diameter $\leq 4^{n+1} d$. We only have to apply Lemma 7.9 to the set $\widetilde{R} \cap E_{0}$. Indeed, take a square $Q$ with diameter $\leq \operatorname{diam}\left(\widetilde{R} \cap E_{0}\right) / 5$. By the induction hypothesis we have

$$
\gamma^{h}\left(Q \cap \widetilde{R} \cap E_{0}\right) \leq A_{0} \gamma_{+}^{h}\left(Q \cap \widetilde{R} \cap E_{0}\right),
$$

because $Q \cap \widetilde{R}$ is a rectangle with diameter $\leq 4^{n} d$. Therefore,

$$
\gamma^{h}\left(\widetilde{R} \cap E_{0}\right) \leq A_{0} \gamma_{+}^{h}\left(\widetilde{R} \cap E_{0}\right)
$$

by Lemma 7.9 . 


\section{REFERENCES}

[Ch] M. Christ, A T(b) theorem with remarks on analytic capacity and the Cauchy Integral. Colloq. Math. 60/61(2) (1990), 601-628.

[Da] G. David. Analytic capacity, Calderón-Zygmund operators, and rectifiability. Publ. Mat. 43 (1999), 3-25.

[Dve] A.M. Davie. Analytic capacity and approximation problems. Trans. Amer. Math. Soc. 171 (1972), 409-444.

[DØ] A.M. Davie and B. Øksendal. Analytic capacity and differentiability properties of finely harmonic functions. Acta Math. 149 (1982),127-152.

[Gar] J. Garnett. Analytic capacity and measure. Lecture Notes in Math. 297, SpringerVerlag, 1972.

[MTV1] J. Mateu, X. Tolsa and J. Verdera. The planar Cantor sets of zero analytic capacity and the local T(b)-Theorem. Preprint (2001). J. Amer. Math. Soc. 16 (2003), 19-28.

[MTV2] J. Mateu, X. Tolsa and J. Verdera. On the semiadditivity of analytic capacity and planar cantor sets. Preprint (2002). To appear in Contemporary Math.

[Ma] P. Mattila. Geometry of sets and measures in Euclidean spaces. Cambridge Stud. Adv. Math. 44, Cambridge Univ. Press, Cambridge, 1995.

[Me1] M.S. Melnikov. Estimate of the Cauchy integral over an analytic curve. (Russian) Mat. Sb. 71(113) (1966), 503-514. Amer. Math. Soc. Translation 80(2) (1969),243256.

[Me2] M.S. Melnikov. Analytic capacity: discrete approach and curvature of a measure. Sbornik: Mathematics 186(6) (1995), 827-846.

[MV] M.S. Melnikov and J. Verdera. A geometric proof of the $L^{2}$ boundedness of the Cauchy integral on Lipschitz graphs. Internat. Math. Res. Notices (1995), 325331.

[NTV1] F. Nazarov, S. Treil and A. Volberg. Weak type estimates and Cotlar inequalities for Calderón-Zygmund operators in nonhomogeneous spaces. Int. Math. Res. Not. 9 (1998), 463-487.

[NTV2] F. Nazarov, S. Treil and A. Volberg. How to prove Vitushkin's conjecture by pulling ourselves up by the hair. Preprint (2000).

[To1] X. Tolsa. Cotlar's inequality and existence of principal values for the Cauchy integral without the doubling condition. J. Reine Angew Math. 502 (1998), 199235.

[To2] X. Tolsa. $L^{2}$-boundedness of the Cauchy integral operator for continuous measures. Duke Math. J. 98(2) (1999), 269-304.

[To3] X. Tolsa. A proof of the weak $(1,1)$ inequality for singular integrals with non doubling measures based on a Calderón-Zygmund decomposition. Publ. Mat. 45:1 (2001), 163-174.

[To4] X. Tolsa. On the analytic capacity $\gamma_{+}$. Indiana Univ. Math. J. 51(2) (2002), 317344.

[To5] X. Tolsa. Painlevé's problem and the semiadditivity of analytic capacity. Preprint (2001). To appear in Acta Math.

[Ve1] J. Verdera. Removability, capacity and approximation. In "Complex Potential Theory", (Montreal, PQ, 1993), NATO Adv. Sci. Int. Ser. C Math. Phys. Sci. 439, Kluwer Academic Publ., Dordrecht, 1994, pp. 419-473.

[Ve2] J. Verdera. On the T(1)-theorem for the Cauchy integral. Ark. Mat. 38 (2000), 183-199.

[VMP] J. Verdera, M.S. Melnikov, and P.V. Paramonov, $C^{1}$-approximation and extension of subharmonic functions, Sbornik: Mathematics 192:4, 515-535. 
[Vi] A. G. Vitushkin, The analytic capacity of sets in problems of approximation theory. Uspeikhi Mat. Nauk. 22(6) (1967), 141-199 (Russian); in Russian Math. Surveys 22 (1967), 139-200.

[VM] A. G. Vitushkin and M. S. Melnikov. Analytic capacity and rational approximation, Linear and complex analysis, Problem book, Lecture Notes in Math. 1403, Springer-Verlag, Berlin, 1984.

Departament de Matemàtiques, Universitat Autònoma de Barcelona, 08193 Bellaterra (Barcelona), Spain

E-mail address: xtolsa@mat.uab.es 